\title{
How much entropy is contained in NMR relaxation parameters?
}

\author{
Falk Hoffmann, ${ }^{*, \dagger}$, Frans A. A. Mulder, ${ }^{*}$, and Lars V. Schäfer ${ }^{*, \dagger}$ \\ $\dagger$ Center for Theoretical Chemistry, Ruhr University Bochum, D-44780 Bochum, Germany \\ $\ddagger$ Current address: Peptone Inc., Northcliff House, Young Street, London, England W8 5, \\ U.K. \\ IInterdisciplinary Nanoscience Center (iNANO) and Department of Chemistry, University \\ of Aarhus, DK-8000 Aarhus, Denmark \\ E-mail: falk@peptone.io; fmulder@chem.au.dk; lars.schaefer@ruhr-uni-bochum.de \\ Phone: +49 (0)234 3221582
}

\begin{abstract}
Solution-state NMR relaxation experiments are the cornerstone to study internal protein dynamics at atomic resolution on time scales that are faster than the overall rotational tumbling time, $\tau_{R}$. Since the motions described by NMR relaxation parameters are connected to thermodynamic quantities like conformational entropies, the question arises how much of the total entropy is contained within this tumbling time. Using all-atom molecular dynamics (MD) simulations of T4 lysozyme, we found that entropy build-up is rather fast for the backbone, such that the majority of the entropy is indeed contained in the short-time dynamics. In contrast, the contribution of slow dynamics of side chains on time scales beyond $\tau_{R}$ on the side chain conformational entropy is significant and should be taken into account for the extraction of accurate thermodynamic properties.
\end{abstract}




\section{Introduction}

Molecular recognition between biomolecules guides many biological processes. The enthalpic contribution to the free energy can often be understood based on minimum-energy structures and plays a fundamental role in computer-aided drug design. ${ }^{1}$ However, the entropic contribution to the free energy is more difficult to understand at the atomic level and is the topic of intense investigation. ${ }^{2-13}$

For a protein in solution, both solvent and protein entropy play an important role for the thermodynamics of the system. In pharmaceutical drug design, one is often interested in site-specific modulations that change the conformational entropy of the protein and/or the ligand. It has been proposed that amide and methyl order parameters from NMR relaxation experiments, which report on the dynamics of protein bonds on ps to ns time scales, can be used as proxies for the backbone and side chain conformational entropy changes in molecular recognition and binding events. ${ }^{14-16}$ Although methyl groups are present only in 6 of the 20 genetically encoded amino acid types in a protein, i.e., residues I, L, V, M, A, T, changes in methyl order parameters provide a reasonably good estimator for the total side chain entropy change of a protein. ${ }^{14}$ While the correlation between NMR order parameter changes and conformational entropy changes is promising, obtaining a perfect correlation presupposes that NMR order parameters report on the full dynamics that are responsible for the change in conformational entropy. Unfortunately, dynamics on longer time scales than the global rotational (tumbling) time of the protein, which is about $10 \mathrm{~ns}$ for a $20 \mathrm{kDa}$ globular protein in aqueous solution at room temperature, do not contribute to relaxation in solution. ${ }^{17}$ It is therefore of interest to quantify the contribution of conformational entropy that is accessible to NMR relaxation with respect to the full conformational entropy.

On the computational side, determining the absolute entropy requires in principle counting of all microstates of a system. While this can be possible for small systems, the exceedingly large number of states in large molecules with many degrees of freedom usually prohibits to directly calculate the complete conformational entropy via counting. For com- 
plex systems like proteins, the configuration space is therefore usually discretized into coarse bins. If the dynamics inside a bin contribute equally among all bins to the full entropy and the time scale of the intra-bin dynamics is faster than the dynamics of changes between different bins, the contribution from intra-bin dynamics can be estimated as an offset to the conformational entropy from the inter-bin dynamics. While such coarse graining of phase space renders the estimation of the conformational entropy practically feasible, it is inherently connected to a loss of information. Therefore, it is necessary to ensure that motions sampled from transitions between coarse-grained bins are still representative for motions of the full system. In the case of protein dynamics, suitable degrees of freedom for the backbone and side chain conformational entropy are the corresponding backbone $(\phi, \psi)$ and side chain $\left(\chi_{i}\right)$ dihedral angles, respectively. Conformational transitions in the protein backbone are directly connected to changes in backbone dihedral angles. Side chains undergo jumps around dihedral angles to interconvert between distinct rotamer states, which can be used for coarse graining the side chain conformational space. These rotamer jumps can occur on a broad range of time scales and also involve rare transitions (slow dynamics) on nanoseconds and beyond. Other side chain motions are usually much faster, such as bond vibrations, angle librations, dihedral vibrations within a single rotamer well, and rotations of methyl groups around their 3-fold symmetry axis.

In the interpretation of experimental NMR relaxation rates, librational motions are widely described by a diffusion-in-a-cone model. ${ }^{18,19}$ With such a simple analytical model, (changes of) NMR relaxation parameters, especially generalized order parameter $S^{2}$, can be transformed into conformational entropy changes, e.g., due to an amino acid mutation or ligand binding. ${ }^{2,20-23}$ Order parameters of rigid bonds in proteins, such as amide $\mathrm{N}-\mathrm{H}$ bonds in well-structured regions, can usually be obtained with high accuracy from MD simulations. However, bonds undergoing transitions between states with long residence times, as is the case for many methyl $\mathrm{C}-\mathrm{H}$ bonds in protein side chains, are more challenging. ${ }^{24-27}$ Low methyl order parameters are connected to the population of several side chain rotamer states, 
which interconvert on a broad range of time scales. ${ }^{25,28-30}$ Slow internal dynamics could also occur for the protein backbone, e.g., in loop regions or (intrinsically) disordered parts. ${ }^{31-33}$ Studies from the Wand lab ${ }^{14-16}$ led to the development of an empirical "entropy meter", in which the change in order parameters derived from NMR relaxation experiments is used to estimate the change in conformational entropy. While these approaches are promising and widely used, they can - by construction - only report on the entropy contributions due to motions that can contribute to NMR relaxation, i.e., within time scales that are faster than the rotational tumbling time of the protein. In an alternate approach, Li and Brüschweiler ${ }^{34}$ created a dictionary of protein side chain entropies from NMR relaxation parameters. It was found that types of amino acids can be grouped together, where the conformational entropy of members of each group correlate similarly to methyl order parameter, but different relations are found between different groups. The effect of dihedral angle correlations on the change of the conformational entropy upon binding is small if the type of motion, e.g., rotameric jumps, is unaffected. ${ }^{35}$ This suggests that (changes in) protein entropy can be estimated from a sum of local contributions, although the calibration of the dictionary may vary from protein to protein. ${ }^{36}$ Using a maximum information spanning tree (MIST) approximation, Fleck and Zagrovic ${ }^{37}$ showed that access to the internal correlation function would be adequate to predict entropy, either from backbone or methyl side chain vectors, and showed that the latter two quantities are also strongly correlated, lending ample support for deriving entropy from NMR dynamics.

We elucidate here how dynamics taking place on various time scales influence the estimation of conformational entropy. MD simulations of T4 lysozyme (T4L) in solution were carried out and the amount of conformational entropy assessed in the simulations due to atomic motions on different timescales was analyzed. Two modern biomolecular force fields were used, namely AMBER ff99SB*-ILDN ${ }^{38,39}$ and CHARMM $36,{ }^{40}$ in each case with reparametrized energy barriers for rotation of methyl groups. ${ }^{24,26}$ It is shown that the majority of the T4L backbone entropy is linked to motions that are faster than the global tumbling 
time of the protein and should hence be accessible to NMR relaxation experiments in solution. In addition, motions within single rotamer wells are sampled on ps time scales, i.e., much faster than the tumbling time of the protein, although slow motions are also present for many methyl-bearing side chains. Thus, entropy contributions arising from infrequent side chain dihedral angle jumps are incompletely captured by classical NMR relaxation experiments. As a consequence, entropy calibration is sensitive to the protein rotational diffusion time, and thereby to protein size and temperature.

\section{Methods}

\section{Simulation Setup}

The setup of the simulations of wildtype T4L with the AMBER force field was described previously. ${ }^{25}$ In brief, the protein was placed in a periodic truncated dodecahedron simulation cell with a minimum distance between protein and the box boundary of initially $1.2 \mathrm{~nm}$, and solvated by adding hydrogens to the crystal waters and 12081 additional TIP4P $/ 2005^{41}$ water molecules. For the simulations with the CHARMM36 force field, the protein was centered in a periodic cubic box with an edge length of $8.0 \mathrm{~nm}$, and solvated by adding hydrogens to the crystal waters and additional 15979 TIP3P water molecules (the CHARMM-specific TIP3Pm water model was used). Sodium and chloride ions were added to obtain neutral simulation systems at a salt concentration of $c=0.15 \mathrm{~mol} / \mathrm{l}$.

\section{Simulations}

All MD simulations were carried out with GROMACS (version 5.0.6). ${ }^{42}$ First, the systems were energy minimized and then equilibrated for 200 ps in the NpT ensemble with harmonic position restraints on all protein heavy atoms (with force constants of $1000 \mathrm{~kJ} \mathrm{~mol}^{-1} \mathrm{~nm}^{-2}$ ). To keep the temperature constant at $300 \mathrm{~K}$, the velocity rescaling thermostat of Bussi and coworkers ${ }^{43}$ was applied, with a coupling time constant of $\tau_{T}=0.1$ ps. Isotropic Parrinello- 
Rahman coupling was used to maintain 1 bar pressure, with a 2 ps coupling time constant and a compressibility of $4.5 \times 10^{-5}$ bar $^{-1}$. The SETTLE ${ }^{44}$ and LINCS ${ }^{45}$ constraint algorithms were applied to constrain the internal degrees of freedom of water molecules and the bond lengths in the protein, respectively, allowing to integrate the equations of motion with $2 \mathrm{fs}$ time steps. The cutoff for the short range Coulomb and Lennard-Jones 12-6 interactions was set to $1.0 \mathrm{~nm}$. Analytical dispersion corrections were added to energy and pressure to correct for the truncation of the Lennard-Jones interactions. Long-range Coulomb interactions were treated with the smooth particle-mesh Ewald (PME) method ${ }^{46}$ with a $0.12 \mathrm{~nm}$ grid spacing.

The equilibrated configurations were used as starting structures for the subsequent production simulations, during which the position restraints were switched off. Ten individual simulations of length $300 \mathrm{ns,}$ each initialized with different atomic velocities, were carried out. Coordinates were saved to disk every 1 ps. Figure S1 shows that the 1 ps time spacing used to store the trajectory frames to disk suffices to reliably extract the amount of conformational entropy from the simulation trajectories already at fast time scales of about 0.5 to $1 \mathrm{~ns}$.

\section{Conformational entropy}

The quasi-harmonic approximation (QHA) as formulated by Schlitter ${ }^{47}$ was used to estimate the backbone conformational entropy from the atomic fluctuations. The QHA provides an upper bound to the true entropy,

$$
S_{\text {conf }}=\frac{k_{B}}{2} \ln \operatorname{det}\left(\mathbf{1}+k_{B} T e^{2} \hbar^{-2} \boldsymbol{M}^{\mathbf{1 / 2}} \boldsymbol{C} \boldsymbol{M}^{\mathbf{1 / 2}}\right) \geq S_{\text {true }},
$$

where $k_{B}$ is the Boltzmann constant, $T$ is the temperature, $e$ is Euler's number, $\hbar$ is the reduced Planck constant, and $\boldsymbol{M}$ is the $3 \mathrm{~N}$-dimensional diagonal mass matrix of the $N$ 
particles. $\boldsymbol{C}$ is the covariance matrix of particle positions,

$$
\boldsymbol{C}=\left\langle(\vec{x}-\langle\vec{x}\rangle)(\vec{x}-\langle\vec{x}\rangle)^{T}\right\rangle
$$

where the $3 N$-dimensional vector $\vec{x}$ represents the Cartesian coordinates of the $N$ backbone $C_{\alpha}$-atoms for which the entropy was calculated. The initial configuration was used as a reference structure for a least-squares fit of trajectory configurations to remove overall translational and rotational motion. The averaging, indicated by $\langle\cdots\rangle$, was carried out over all trajectory frames up to a certain time $t$, thus yielding the entropy build-up over time.

To calculate backbone and side chain entropies on a per-residue basis, backbone $\phi$ and $\psi$ dihedral angles and side chain $\chi$ dihedral angles were extracted from the MD trajectories and binned in increments of $18^{\circ}$ and $120^{\circ}$ for $\phi / \psi$ and $\chi$, respectively. For the side chain $\chi$ dihedral angles, the discretization was chosen such as to represent the three main rotamer wells trans, gauche ${ }^{+}$, and gauche ${ }^{-}{ }^{48}$ Additional analyses with $3^{\circ}$ binning of $\chi$ were also carried out. For each backbone group, the two-dimensional (2D) $\phi / \psi$ histogram was accumulated, and the 2D joint probability distribution $P_{b b}(\phi, \psi)$ was computed. Similarly, a rotamer probability distribution $P_{s c}(\chi)$ of all $3^{N_{\chi}}$ rotamers of each side chain was constructed from the $\chi$-angle histograms, where $N_{\chi}$ is the number of side chain $\chi$ dihedral angles. The backbone and side chain entropies $S_{b b}$ and $S_{s c}$ were calculated according to

$$
\begin{aligned}
& S_{b b}=-k_{B} \sum_{i=1}^{20} \sum_{j=1}^{20} P_{b b}\left(\phi_{i}, \psi_{j}\right) \ln P_{b b}\left(\phi_{i}, \psi_{j}\right) \text { and } \\
& S_{s c}=-k_{B} / N_{\chi} \sum_{i=1}^{3^{N} \chi} P_{s c}\left(\chi_{i}\right) \ln P_{s c}\left(\chi_{i}\right),
\end{aligned}
$$

respectively, where the sum is over all bins $i$ and $j$ for the backbone, or over all rotamer wells of the $N_{\chi}$ side chain dihedral angles. The side chain entropies were normalized to the number of $\chi$-angles in the respective side chain $N_{\chi}$. As the absolute value of $S_{\text {conf }}$ is not of primary interest here, we neglect higher order interangle correlations, e.g., due to correlated 
motions between $\phi$ and $\psi$ angles that do not flank the same backbone amide, or between backbone and side chain dihedral angles. Although correlated motions can affect (changes in) conformational entropy, ${ }^{14,49,50}$ they are not expected to significantly change the rate at which the entropy is built up, i.e., the speed at which a certain part of configuration space is explored.

\section{Results and Discussion}

\section{Backbone entropy from quasi-harmonic approximation}

The build-up of the conformational entropy of the T4L backbone was obtained from the Schlitter QHA approach ${ }^{47}$ (Figure 1). The QHA describes collective motions of all backbone $C^{\alpha}$ atoms and provides an upper bound to the true conformational entropy. A principal limitation of QHA is that it overestimates $S_{\text {conf }}$ due to the neglect of anharmonicity (e.g., due to multiple minima) as well as nonlinear and higher-order correlations. However, the focus of the present study is not on accurate absolute values but on the build-up rate, i.e., the relative percentage of entropy that is linked to motions sampled within a certain time scale. While anharmonicities and higher-order correlations affect the absolute value of $S_{\text {conf }}$, they are not expected to have an effect on the build-up rate. Both the AMBER ff99SB*-ILDN and the CHARMM36 force field predict that about $95 \%$ of the total conformational entropy, as obtained from the complete aggregated simulation time of $3 \mu s$, is already sampled within

the tumbling time $\tau_{R}$, which is about $11 \mathrm{~ns}$ for T4L. ${ }^{25}$ The entropy build-up profile is highly comparable for the two force fields. 

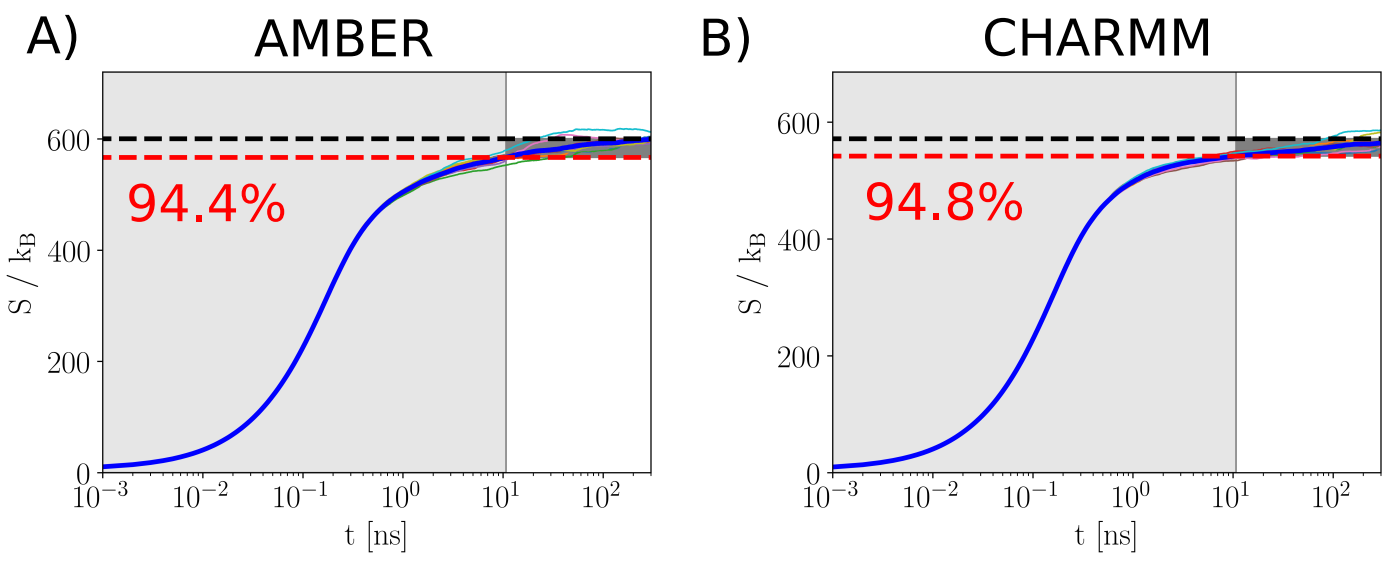

Figure 1: Cumulative conformational entropy of the T4L backbone, calculated with the Schlitter quasi-harmonic approximation, in the MD simulations with the AMBER ff99SB*ILDN (A) and the CHARMM36 (B) force field. The thick blue line shows the average, while the thin lines show the data from the 10 individual simulations. The light gray area represents the protein tumbling time $t_{a} u_{R}$, which is about $11 \mathrm{~ns}$ for T4L in aqueous solution at room temperature. ${ }^{25}$ The dark gray area displays the conformational entropy due to motions beyond $t \leq \tau_{R}$, which is sampled in MD but is not accessible in NMR relaxation experiments in solution. The red and black dashed lines show the average entropy at $\tau_{R}$ and the long-time limit, respectively.

Interestingly, about $90 \%$ of the long-time limit entropy is already sampled within $1 \mathrm{~ns}$, which is faster than the tumbling time of any folded protein in aqueous solution at ambient conditions. This means that the majority of the thermodynamic information related to backbone motion is contained in NMR relaxation data, although about $5 \%$ of the conformational entropy is due to slow motions and can only be detected on time scales longer than $\tau_{R}$.

\section{Single-residue entropies: backbone}

In an NMR relaxation experiment, one usually measures the dynamics of a local bond or interaction, and order parameters $S^{2}$ describe the motional restriction of a specific bond. The aim of an entropy meter is to quantitatively connect these local dynamic probes to conformational entropies, and single-residue conformational entropies were calculated from the distributions of the corresponding backbone or side chain dihedral angles (see Eqs. 3 and 4). The configurational entropies at the tumbling time and in the long-time limit are 
provided in Tables S1 and S2 for all 158 non-proline residues of T4L (except MET1), and are shown in Figure 2 for the backbone and in Figure 3 for the protein side-chains, respectively.
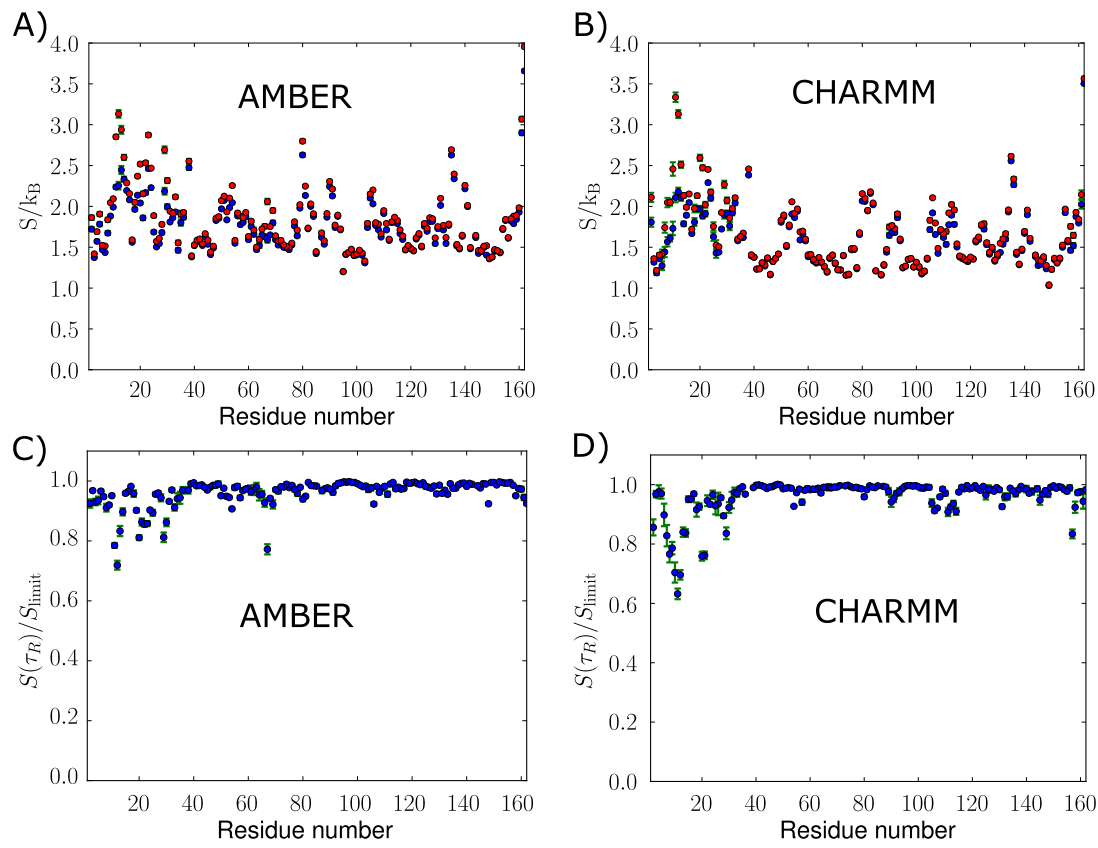

Figure 2: Conformational entropy sampled within the tumbling time $\tau_{R}$ (blue data points) and in the long-time limit (red data points) for the T4L backbone in the MD simulations with the AMBER (A) and CHARMM (B) force field. The ratio of the conformational entropies sampled within $\tau_{R}$ and in the long-time limit are plotted in panels $\mathrm{C}$ ) and $\mathrm{D}$ ). The errors plotted are the standard deviations from the 10 individual MD simulations. 

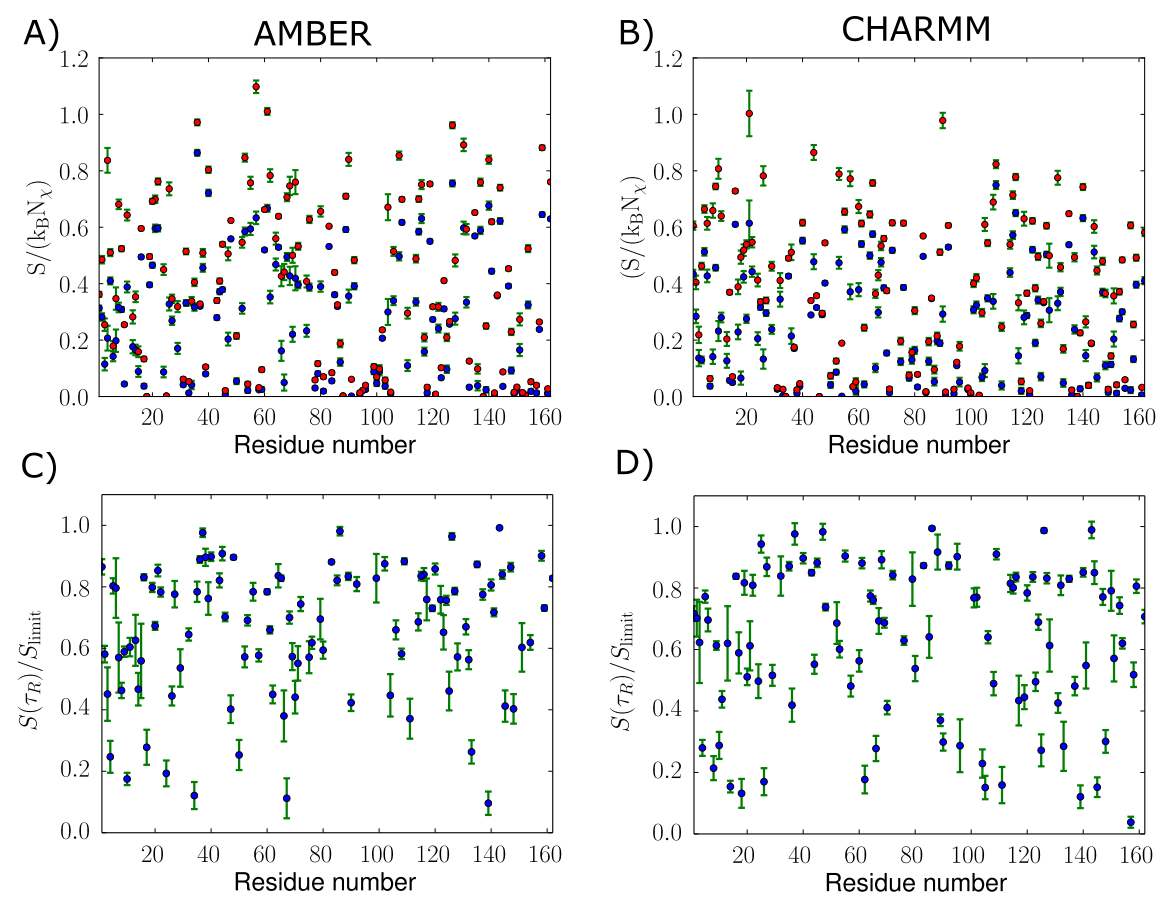

Figure 3: Conformational entropy sampled within the tumbling time $\tau_{R}$ (blue data points) and in the long-time limit (red data points) for side chains with $120^{\circ}$ binning of the dihedral angles describing the side chain rotamer wells. The ratio of the conformational entropies sampled within $\tau_{R}$ and in the long-time limit are plotted in panels C) and D). The errors plotted are the standard deviations from the 10 individual MD simulations.
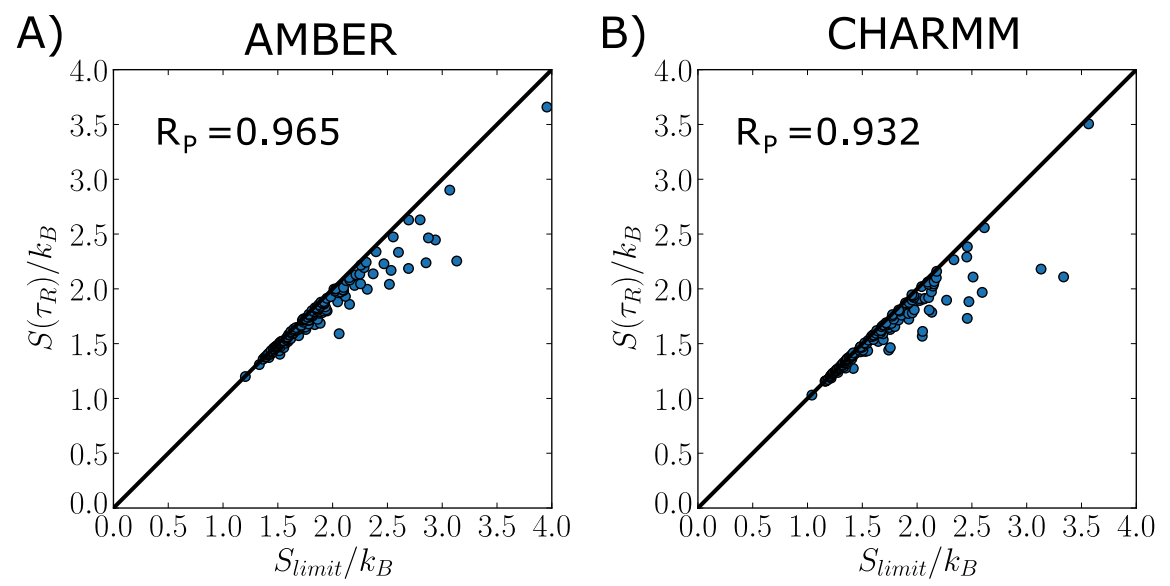

Figure 4: Correlation between conformational entropies sampled after the tumbling time $\tau_{R}$ and in the long-time limit for the T4L backbone in the simulations with the AMBER (A) and CHARMM (B) force field.

The data show that the backbone entropy build-up is rather fast for most residues. For 146 of the 158 residues, more than $90 \%$ of the long-time conformational entropy is already 
reached within the tumbling time in the simulations with the ff99SB*-ILDN force field, and only three residues sample less than $80 \%$ of the long-time conformational entropy, GLY12 (72\%), PHE67 (77\%) and GLU11 (78\%). This result is consistent with the interpretation that fast librational motions of backbone bonds rapidly sample the conformational space within a restricted region of the Ramachandran map. As long as there are no barrier-crossing transitions to alternative metastates, entropy build-up is fast. Except for PHE67, all residues with less than $90 \%$ of the long-time limit conformational entropy are located in the $\beta$-region encompassing residues 10 to 30 (see Figure 2). This region is highly conserved among different lysozyme families and is key to the enzyme's catalytic activity. ${ }^{51}$ Interestingly, the catalytic residues GLU11 and ASP20 are among those with the slowest entropy build-up, suggesting that their motional restriction and structural preorganization may be tuned for enzyme action. Although details vary, a similar overall picture emerges from the simulations performed with the CHARMM force field (Figure S2). The entropy build-up is slightly slower with the CHARMM36 force field than with AMBER ff99SB*-ILDN (see Tables S1 and S2 in the SI). Especially for some residues close to the active site, the entropy build-up rate is apparently lower for CHARMM36 than for AMBER ff99SB*-ILDN. This could indicate that the energy landscape of CHARMM36 is more rugged, a point that might warrant further investigations. ${ }^{52}$

Even so, Figure 4 shows that there is a good correlation between the backbone configurational entropies sampled at $\tau_{R}$ and in the long-time limit, with Pearson correlation coefficients of 0.97 and 0.93 for AMBER and CHARMM, respectively. This shows that a simple relation between relaxation parameters and entropies, which underpins an empirical entropy meter, can be expected to work well for backbone motions, because the estimated entropies on short time scales are highly correlated to the full configurational entropies.

Figure 5 shows the entropy build-up of those T4L residues that exhibit the lowest and highest amount of backbone conformational entropy sampled at the tumbling time relative to the long-time limit. The sampling of the conformational backbone entropy with a binning 
of $18^{\circ}$ for the backbone dihedral angles $\phi$ and $\psi$ contains motions within core regions of the Ramachandran map, e.g., within the $\alpha$ helical region, which are fast. This is in line with the notion that backbone motions in structured regions comprise of librational motions on ps time scales. On the other hand, conformational changes involving barrier crossings between regions, e.g., a helix-coil transition, happen on much longer time scales and would lead to a slower entropy build-up. This did not occur in our simulations of T4 lysozyme.
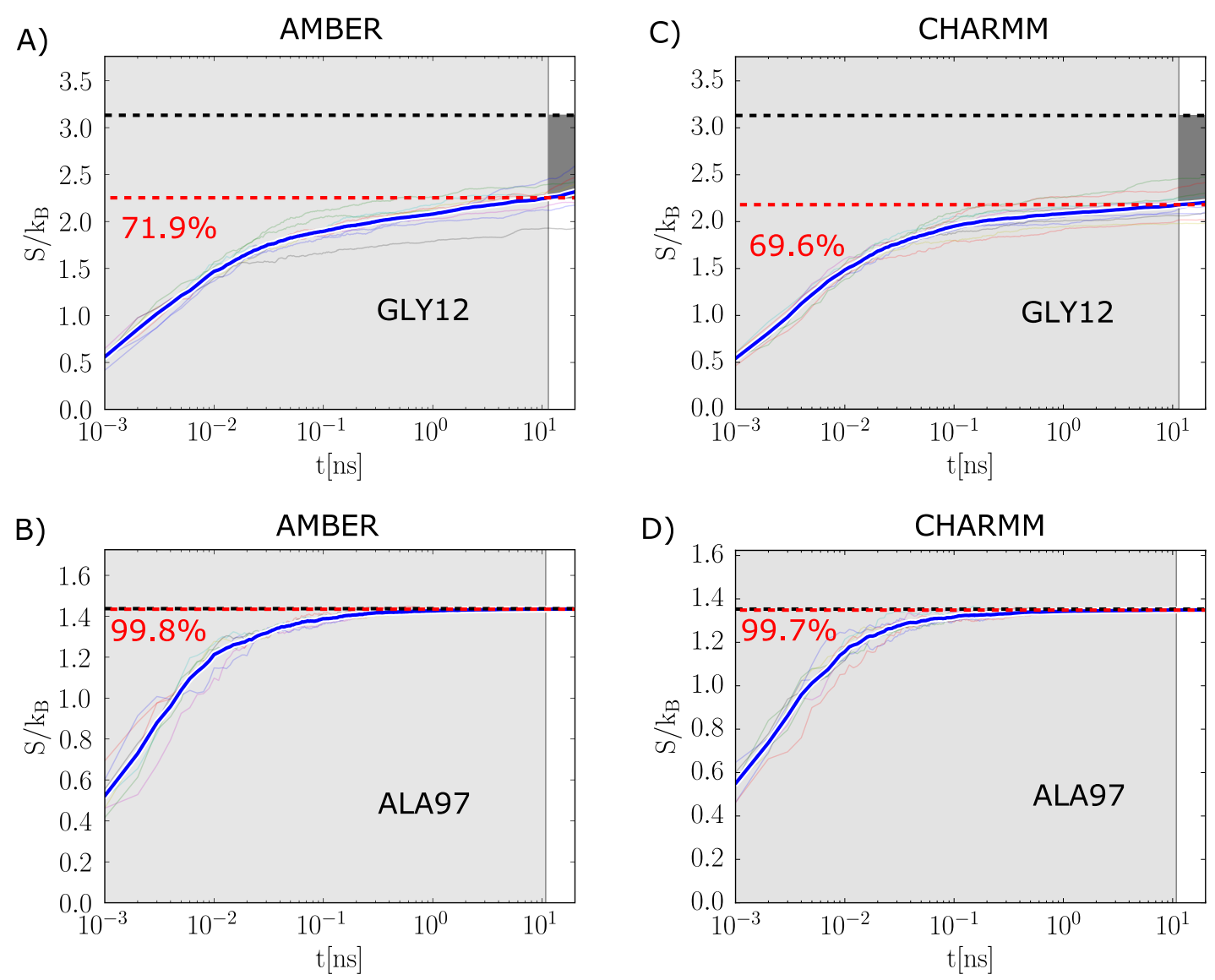

Figure 5: Backbone entropy build-up of residues GLY12 (top) and ALA97 (bottom) in the simulations with the AMBER (A and B) and CHARMM (C and D) force fields. The results show an average of 15 non-overlapping $20 \mathrm{~ns}$ blocks for every of the 10 individual 300-ns simulations (i.e., 150 blocks in total). The thin colored lines and the thick blue line represent the entropy build-up of the 10 different simulations and their average, respectively. The red and black dashed lines show the averaged entropy at $\tau_{R}$ and the long-time limit entropy (after $3 \mu$ s accumulated sampling), respectively. 


\section{Single-residue entropies: side chains}

Next, the residual conformational entropy of the side chains was investigated. Figure 3 shows the side chain configurational entropies sampled within the tumbling time and in the long time limit for all side chains with a $120^{\circ}$ binning of the dihedrals. Figure $6 \mathrm{~A}$ and $\mathrm{B}$ show the correlation between these two entropy values. The individual values of all side chains and both force fields can be found in Tables S3 and S4 in the Supporting Information.
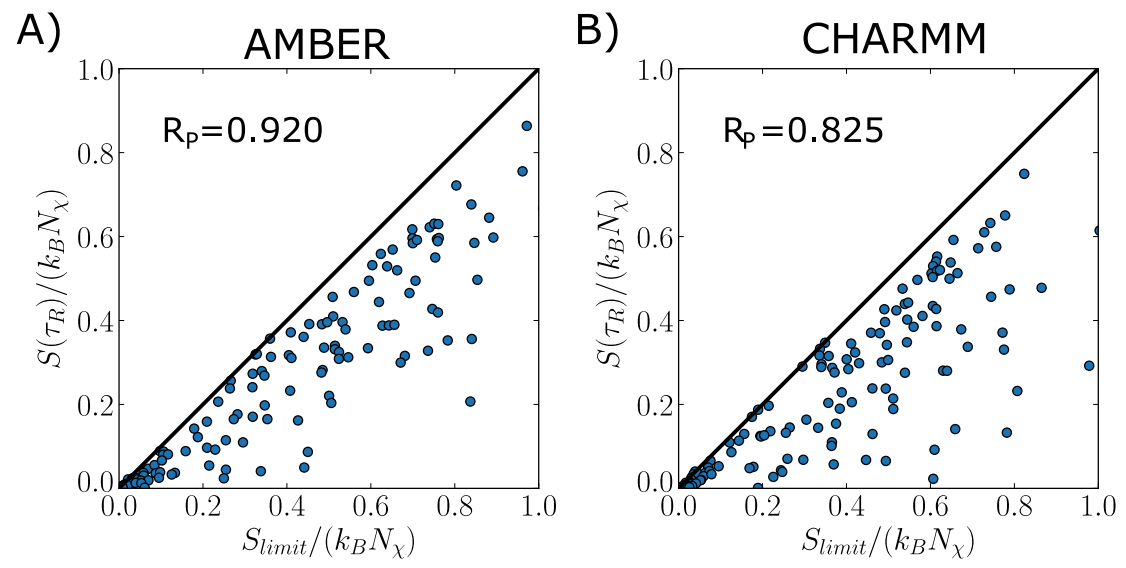

Figure 6: Correlation between conformational entropies sampled after the tumbling time $\tau_{R}$ and in the long-time limit for side chains with $120^{\circ}$ binning for the $\operatorname{AMBER}(\mathrm{A})$ and CHARMM (B) force fields.

The comparison between backbone and side chain conformational entropies with $120^{\circ}$ bins shows that variations in the backbone entropy are more closely linked to specific parts of the protein than is the case for the side chains. For example, 31 of the 36 residues with a long-time limit backbone conformational entropy of more than $2 k_{\mathrm{B}}$ have at least one neighbouring residue that also has a long-time limit backbone entropy of more than $2 k_{\mathrm{B}}$. For the backbone, the motions of a particular dihedral angle are coupled to the neighboring backbone dihedrals. This is not the case for side chains, where dynamic couplings between consecutive dihedral angles are not observed (Figure $3 \mathrm{~A}$ and $\mathrm{B}$ ). While backbone dihedral angles of neighboring residues are directly connected along the sequence, side chain dihedral angles of neighboring residues are spatially and morphologically separated and thus move 
largely independent of each other. The motions of these side chains are dominated by fast fluctuations within individual rotamer states and slower jumps between these.

Next, it was investigated how much of the conformational entropy due to intra-rotamer motions is sampled within the tumbling time. Figure 7 shows that with a $3^{\circ}$ binning the entropy build-up is nearly completed within the tumbling time, with a high correlation between short- and long-time entropy $\left(R_{P}=0.95\right)$, similar to the backbone motions. In contrast, Figure 6 A shows that a significant amount of long-time limit side chain conformational entropy is not sampled within the tumbling time for the same force field with a $120^{\circ}$ binning, i.e., if only the jumps between the main rotamer states (and not the intra-well motions) are considered. This means that the intra-rotamer well fluctuations are sampled within the tumbling time, but that a significant amount of inter-rotamer motion occurs on slower time scales. The amount of missing conformational entropy at $\tau_{R}$ that results from changes in the width of the rotamer wells is small in comparison with the conformational entropy contribution due to jumps between distinct rotamer wells.
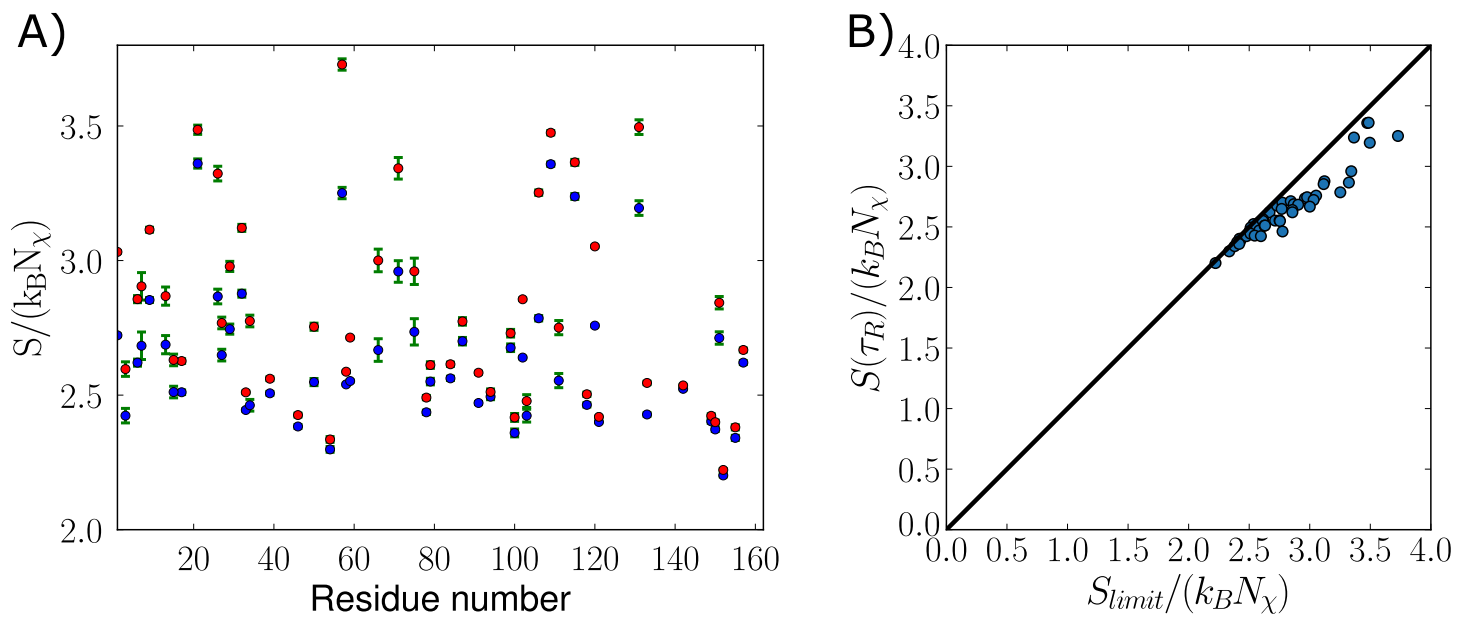

Figure 7: A) Absolute conformational entropy for side chains with $3^{\circ}$ binning, sampled within the tumbling time $\tau_{R}$ and in the long-time limit (blue and red data points, respectively), and $\mathrm{B})$ the correlation between the two.

We further investigated the missing side chain conformational entropy and estimated the percentage of side chains with a fast and slow entropy build-up rate. All side chains that do 
not deviate much from the regression line in Figure 7 B show a fast entropy build-up. Using THR142 as a representative example, it is seen that more than $90 \%$ of the long-time side chain conformational entropy is sampled already within less than 100 ps (Figure 8). Such a fast entropy build-up is a result of the rapid exploration of the intra-rotamer phase space if the mean residence time within the rotamer wells is long. Although it does not mean that there are no jumps to other rotamer states with short residence times, but that these are rare and hardly populated the gauche ${ }^{-}$state of the $\chi_{1}$ dihedral angle for THR142 (Figure 8 E) and therefore do not contribute significantly to the total conformational entropy.

About $20 \%$ of residues in T4L have a long-time limit side chain conformational entropy of less than $0.2 k_{\mathrm{B}} N_{\chi}$. These side chains display very limited rotamer jump dynamics on the sub$\mu$ s time scale and effectively occupy a single dominant rotamer state. Their contribution to the total side chain rotamer entropy is small in comparison to other residues. The dynamics of these side chains is dominated by fast librational motions within single rotamer wells on ps time scales.

We next focus on those side chains in T4L for which less than $90 \%$ of the long-time limit conformational entropy is sampled within the tumbling time. These display a slow build-up rate, and contain at least one rotamer state that is frequently visited with very short residence times during the MD simulations. As an example, Figure $8 \mathrm{~F}$ shows the time evolution for $\chi_{1}$ of MET106 during a representative trajectory. There are frequent transitions from and to the trans rotamer, but this state is only populated for few consecutive ps.

The entropy build-up rate associated with intra-rotamer well motions is fast if all states relevant to the total conformational entropy have a mean residence time long enough to explore the intra-rotamer well phase space, which is the case for most occupied rotamer wells of protein side chains. Furthermore, we record the fact that the contribution of intrarotamer motions to the conformational entropy does not change among different rotamer wells and can be seen as an offset $S_{\text {intra }}$ to the total conformational side chain entropy, $S=S_{\text {inter }}+N_{\chi} \cdot S_{\text {intra }}$. 
Next, we focus on the slower and hence more challenging inter-rotamer well motions and the related entropy contributions $S_{\text {inter }}$. These are counted by jump transitions between distinct rotamer wells with bins of size $120^{\circ}$. For residues with a long-time limit side chain conformational entropy larger than $0.2 k_{\mathrm{B}} N_{\chi}$ in the AMBER ff99SB*-ILDN simulations, less than $90 \%$ of the long-time limit entropy was sampled within $\tau_{R}$ for most side chains (Figure 3 C and D), showing that the desired equilibrium propensity of all rotamer states is not fully sampled within the tumbling time of the protein. 

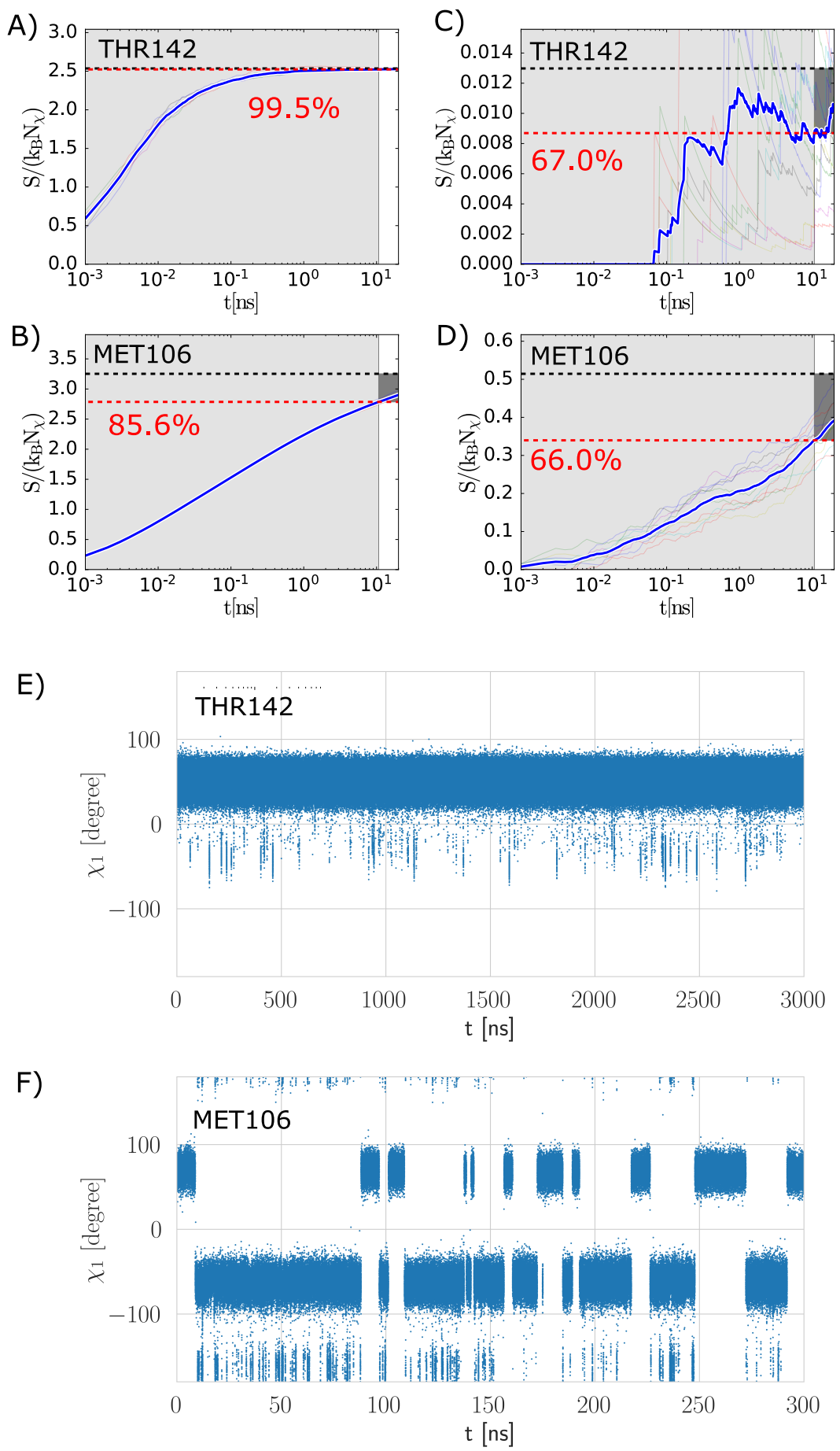

Figure 8: Side chain entropy build-up with $3^{\circ}$ bins (A, B) and with $120^{\circ}$ bins (C, D) of residues THR142 (top) and MET106 (bottom). Panels E) and F) show representative trajectories of the side chain dihedral angle $\chi_{1}$ in THR142 (E) and MET106 (F). The thin lines and the thick blue line represent the entropy build-up of the 10 different simulations and their average, respectively. The red and black dashed lines show the entropy at $\tau_{R}$ and the long-time limit entropy after $3 \mu$ s, respectively. 

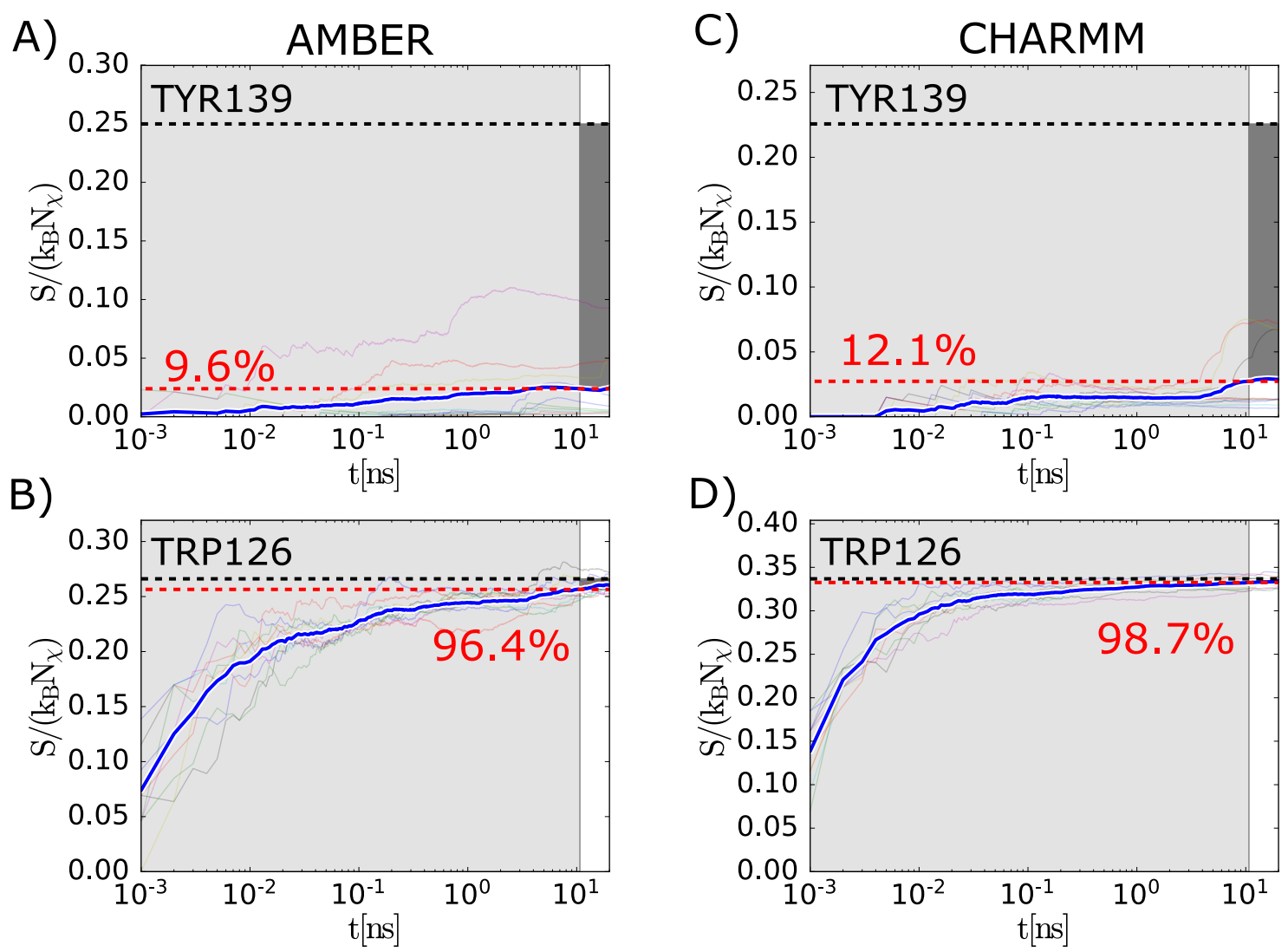

Figure 9: Side chain entropy build-up with $120^{\circ}$ binning of residues TYR139 (top) and TRP126 (bottom) for the AMBER ff99SB*-ILDN (A and B) and CHARMM36 (C and D) force fields. The results show an average of 15 non-overlapping 20 ns blocks per simulation from all 10 simulations. The thin lines and the thick blue line represent the entropy build-up of the 10 individual simulations and their average, respectively. The red and black dashed line show the averaged entropy at $\tau_{R}$ and the long-time limit entropy after $3 \mu \mathrm{s}$, respectively.

Interestingly, the few side chains for which nearly all of the long-time conformational entropy is sampled within the tumbling time are typically aromatic rings. The rigidity of these side chains constrains the available side chain dihedral angle phase space. For example, more than $80 \%$ of the long-time limit side chain conformational entropy of TRP126 is sampled within 100 ps with the AMBER ff99SB*-ILDN force field (Figure 9B), and even already within 10 ps with the CHARMM36 force field (Figure 9D). Rotamer jumps of dihedral angles in aromatic rings are slower than in other side chains. For example, Kulkarni and Söderhjelm ${ }^{53}$ used enhanced sampling techniques to show that the flip rates of the aromatic rings of the 4 tyrosine and the 4 phenylalanine side chains in basic pancreatic trypsin inhibitor 
(BPTI) lie between $280 \mu \mathrm{s}$ and $3.5 \mathrm{~s}$, i.e., they are much slower than the simulation times typically reached in all-atom MD simulations. Such cases can occur, but they are usually not considered in an entropy meter because the minor state contributes only a small amount of the total conformational entropy. Our data show that for most other side chains, rotamer jumps on time scales between $\tau_{R}$ and the MD simulation time are present. The slow entropy build-up for these side chains might point to differences in side chain dynamics among the independent MD simulations. For example, Figures 9A and 9C show that even for TYR139, the side chain with the lowest amount of relative conformational entropy at $\tau_{R}$ with respect to its long-time limit entropy in the AMBER ff99SB*-ILDN force field, all 10 simulations show nonzero side-chain conformational entropy as a consequence of rotamer transitions, but the cumulative conformational entropy as an average over the 15 blocks in a simulation does not exceed $0.2 k_{\mathrm{B}} N_{\chi}$ at $\tau_{R}$ in any of these simulations. However, the cumulative conformational entropy of this side chain from the concatenated trajectory is $0.5 k_{\mathrm{B}} N_{\chi}$, which is 2.5 times higher than their largest individual contribution. This suggests that, even in the presence of transitions between rotamers, not all the phase space is sufficiently sampled within time scales shorter than $\tau_{R}$ and may not be detected by a dynamical observable in an NMR relaxation experiment in solution.

To test this hypothesis, we next look at the side chain dihedral angle motions in more detail. Our above analyses of all methyl groups of T4 lysozyme ${ }^{25}$ showed that LEU66-C ${ }^{\delta, 2}$ is the methyl group with the highest order parameter. One might expect that such high order parameter is connected to a population of a single rotamer well. ${ }^{28,54,55}$ Figure $10 \mathrm{~A}$ shows the side chain dihedral angle $\chi_{2}$ of LEU66 for three representative simulations. The trajectories for all simulations are shown in Figure S3. It can be seen that at least two states are occupied in every simulation, but the mean residence time within the rotamer wells is often longer than $\tau_{R}$, and the distributions from the individual simulations are quite different. 

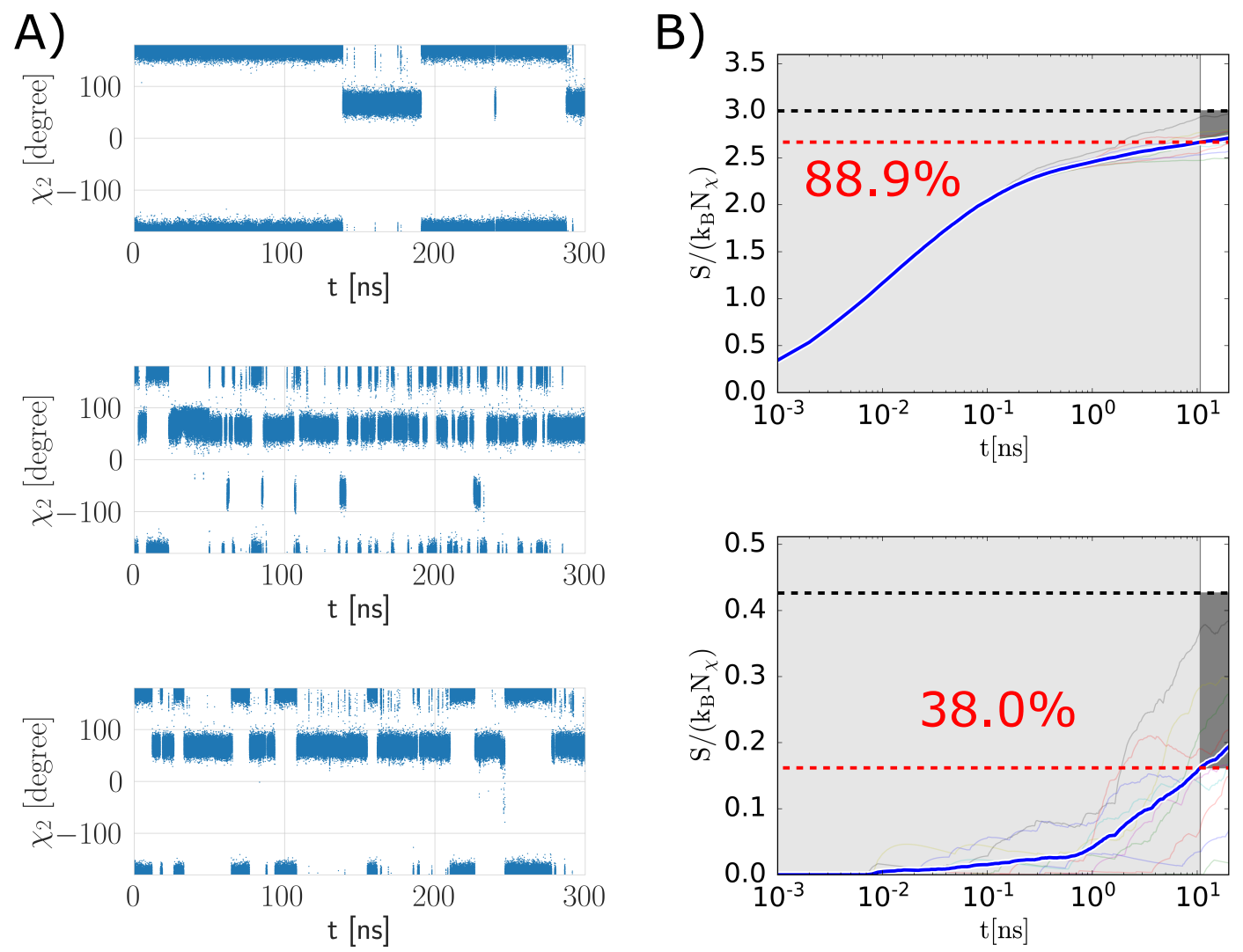

Figure 10: A) Side chain dihedral angle $\chi_{2}$ of LEU66 of three representative simulations. B) Entropy build-up for LEU66 for a $3^{\circ}$ (top) and $120^{\circ}$ (bottom) binning.

In general, different distributions of rotameric states with varying residence times are sampled. Single trajectories can be trapped in a specific region of the potential energy landscape, which might give a misleading interpretation of the frequency of jumps between states and the average residence time within a state. This sampling problem can be reduced somewhat by running multiple shorter simulations. For example, Allnér and coworkers ${ }^{56}$ showed that the number of conformational clusters of backbone dihedral angles sampled within $100 \mathrm{~ns}$ can be doubled if the simulation length is divided into blocks of $10 \times 10 \mathrm{~ns}$ or $5 \times 20$ ns with different initial velocities. However, the sampling in any single trajectory has to be long enough to capture processes on all relevant time scales. Here we chose a simulation length of $300 \mathrm{~ns}$ for every individual simulation, which exceeds the tumbling correlation time of T4L by about a factor of 30 and hence should be long enough to capture motions on time 
scales faster than $\tau_{R}$. The different residence times among the individual simulations show that many side chain rotamer transitions occur infrequently and that the complete sampling of the absolute side chain conformational entropy might require additional and/or longer simulations. For the present study, in which we investigate the rate of entropy build-up over time and not the absolute entropy, the broad distribution of transition frequencies sampled from 10 individual simulations of $300 \mathrm{~ns}$ provides a reasonable estimate.

The frequency of the transitions between rotamer states has a large impact on order parameters $S^{2}$. Frequent transitions on time scales faster than $\tau_{R}$ decrease the TCF and are contained in the experimental NMR relaxation data; few transitions between states with long residence times lead to a similar canonical rotamer distribution in the long-time limit, but do not contribute to the NMR relaxation data because the TCF has already decayed to zero due to overall tumbling. In consequence, contributions to the conformational entropy due to slow rotamer interconversion are usually not present in the relaxation data. Figure 10B shows the side chain entropy build-up of LEU66. One can see that the fast exploration of the inner-rotamer wells leads to a fast entropy build-up with the $3^{\circ}$ binnning, but the entropy build-up with a $120^{\circ}$ binning is indeed relatively slow and only $38 \%$ of the long-time limit entropy with a $120^{\circ}$ binning is sampled up to the tumbling time. Jumps from and to states with long residence times in the trajectories of Figure 10A can be directly connected to the entropy build-up steps of the same trajectories in Figure 10B. Transitions between rotamer states with residence times longer than the tumbling time of the protein are not probed in relaxation experiments, but they can be captured by residual dipolar couplings (RDCs) or J-couplings. For instance, Farès and coworkers ${ }^{57}$ measured NMR order parameters from relaxation experiments and RDC experiments for ubiquitin. They showed that the correlation between the two data sets is reasonable (correlation coefficient of 0.73 ), but that order parameter from relaxation experiments are systematically overestimated because they do not include the effect of "supra- $\tau_{R}$ " motions. We conclude that one has to be cautious if conformational entropies are extracted from side chain NMR relaxation parameters because 
entropy contributions from rotamer transitions between states with long residence times will be missing.

\section{Side chain entropy and order parameter}

In the previous section, the different types of motions that influence the conformational entropy of proteins were discussed. It was found that a significant amount of conformational entropy is missing in NMR order parameters if rotamer jumps on long time scales are present within the side chain. In this section, we address the question how big the influence of this missing entropy might be on an (empirical) entropy meter. Therefore, the side chain conformational entropy of methyl-bearing side chains is compared with the methyl order parameter, $S_{\text {axis }}^{2}$. Figure 11 shows the side chain conformational entropy as a function of the order parameter from the simulation with the AMBER ff99SB*-ILDN force field for all methyl groups. First, we focus on the effect of intra-rotamer well motions on methyl order parameter. Regression lines for entropies at $\tau_{R}$ and in the long-time limit with a binning of $120^{\circ}$ converge for $S=0$ to similar methyl order parameter $S_{\text {axis }}^{2}(S=0) \approx 0.84$ and 0.88, respectively. That means that methyl groups without rotamer jumps $(S=0$ with $120^{\circ}$ binning) display motions within a single rotamer well that decrease the methyl order parameter $S_{\text {axis }}^{2}$ from 1.0 to 0.88 . The conformational entropy with a binning of $3^{\circ}$ for $S_{\text {axis }}^{2}=0.88$ is $S \approx 2.4 k_{B} N_{\chi}$ (Figure 11B). As described above, this entropy can be seen as an offset to the full conformational entropy and is uncoupled from motions between rotamer wells. This result can be illustrated by the THR152 side chain, the methyl group with the slowest rotamer transitions $\left(S=0.014 k_{B} N_{\chi}\right.$ in the long-time limit with $120^{\circ}$ binning),

which indeed has a similar order parameter and $3^{\circ}$ binning entropy $\left(0.87\right.$ and $\left.2.22 k_{B} N_{\chi}\right)$ as estimated from the linear regression. 

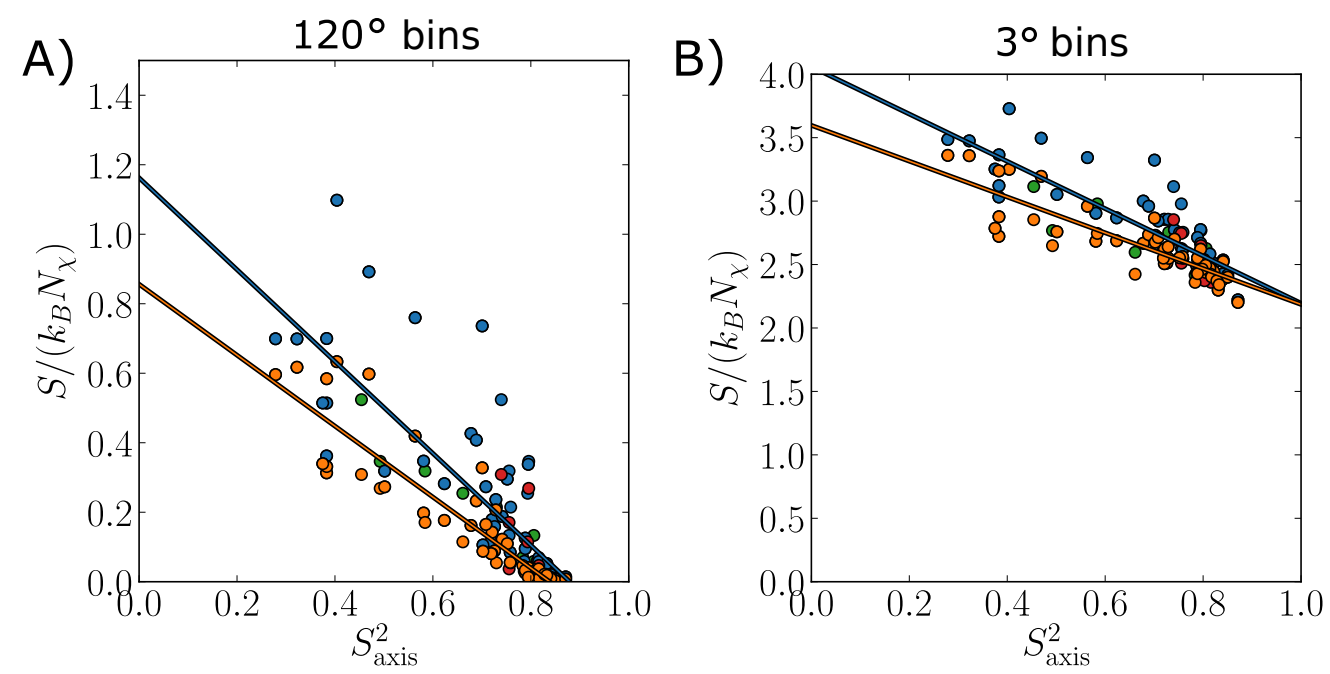

Figure 11: Side chain entropy with $120^{\circ}$ binning (A) and with $3^{\circ}$ binning (B) for all nonalanine methyl groups of T4L as a function of the methyl order parameter $S_{\text {axis }}^{2}$ from the simulations with the AMBER ff99SB*-ILDN force field. The orange and blue dots represent the side chain entropy within the tumbling time and in the long-time limit, respectively. The orange and blue lines are linear regression lines to the respective data. Red and green dots represent the values of the ILE-C ${ }^{\gamma}$ methyl groups that were not included for fitting the regression line. Methyl order parameters of the two methyl groups in VAL and LEU were averaged.

Next, we turn the focus to the influence of rotamer jumps on the entropy meter. An entropy meter describes the parametric relation between conformational entropy from the explored phase space and order parameters as dynamical proxies. If this function is linear, the slope of the regression line describes the entropy meter completely. The slope of the regression line in the long-time limit is about 30\% larger than the slope of the regression line of entropies at $\tau_{R}$. This is the case both for the $3^{\circ}$ and for the $120^{\circ}$ binning. In addition, the ratio depends on the choice of side chain dihedral angles. The spread of the data in Figure 11 suggests that the linear decrease in residual conformational entropy as a function of methyl order parameter does not include the chemical composition of the side chains involved, as was done in other approaches. ${ }^{34}$ Furthermore, the spread of the data around the regression line increases more strongly from $\tau_{R}$ to the long-time limit than the slope does, suggesting that conformational entropy from slow dynamics beyond $\tau_{R}$, which are not captured in methyl order parameter, are not linearly related to methyl order parameter. As described above, 
the additional configurational space not contained in relaxation parameters results from exploring states with residence times beyond $\tau_{R}$. Because the intra-rotamer well dynamics can be seen as an offset (see above), the small amount of additional dynamics from intrarotamer dynamics at long time scales for these kind of motions is not enough to capture the relatively large portion of additional configurational space. The presence of rotamer jumps between states with long mean residence times is therefore linked to higher conformational entropy, without an equal effect on methyl order parameter. Such nonlinear behavior cannot be remedied by adjusting the prefactor of the entropy meter but calls for the implementation of other functional forms. We conclude that the contribution of slow dynamics of side chains on time scales beyond $\tau_{R}$ on the side chain conformational entropy is significant and should be considered for the extraction of accurate thermodynamic properties.

Solution-state NMR relaxation experiments are a valuable tool to investigate protein motions on time scales shorter than the tumbling time $\tau_{R}$ of a protein. Improvements in the accuracy of MD simulations will continue to provide insight into the relationship between NMR order parameters and thermodynamic quantities. Recent studies ${ }^{14,15}$ have established a direct relationship between MD amide and methyl order parameter and backbone and side chain conformational entropies, respectively, based on dihedral angle distributions. Force field improvements, longer simulation times, and proper treatment of anisotropic tumbling can increase the accuracy of MD order parameter with respect to their experimental counterparts. ${ }^{24-27,58}$ In order to increase the sensitivity of solution-state to slower motions, approaches are needed that can drastically slow molecular tumbling, but without increasing the NMR linewidths. Fast and transient exchange with the corona of nanoparticles may constitute one such avenue. ${ }^{59}$ 


\section{Conclusions}

Using T4 lysozyme as a case example, it was shown that almost all of the conformational entropy connected to motions of backbone atoms is sampled within times shorter than the overall tumbling correlation time $\tau_{R}$. Most of the residual conformational backbone entropy in structured regions of T4L result from sampling inside single wells on the Ramachandran map. These individual wells are explored rapidly, and the fast intra-well motions will be reflected in NMR relaxation data. Slow motions observed for residues close to the active site may point to dynamically stable (long-lived) metastable states in this region, with transitions between them on time scales longer than $\tau_{R}$. At the level of individual residues, amino acids with slower backbone entropy build-up are usually consecutive residues in the protein. In particular, the active site of T4L contains residues with a remarkably slow entropy build-up.

Intra-rotamer motions in side chains are sampled very fast and lead to an offset to the total conformational entropy, which is dictated by the number of occupied rotamer wells and the mean residence times within these wells. Intra-rotamer well motions in side chains decrease side chain order parameters from 1.0 to about $0.84-0.88$, while inter-rotamer jumps in side chains lead to a further decrease to lower methyl order parameters and are the main source for the proposed relation between conformational side chain entropy and methyl order parameter in form of an "entropy meter". However, side chain conformational entropies depend on slow jumps between rotamer wells. Our data suggests that the relation depends on the local environment of the side chain, which has a significant influence on the rotamer jump frequency and mean residence time within a rotamer well. Motions slower than the global rotational tumbling time of the protein lead to a conformational entropy that is on average about $30 \%$ higher than the conformational entropy without these motions. We observe that the relation between total conformational entropy and methyl order parameter is nonlinear in comparison to the linear relation between entropies captured on time scales accessible by NMR relaxation parameter in solution and methyl order parameter. We anticipate that the results of this study can help estimate accurate thermodynamic quantities from NMR 
relaxation parameters and guide a way to recalibrate an empirical entropy meter with the help of molecular dynamics simulations.

\section{Supporting Information Available}

QHA entropy buildup analyzed by resampling from the simulations with different time steps. Correlation between backbone entropy at $\tau_{R}$ and in the long-time limit. Time traces of the

$\chi_{2}$ side chain dihedral angle of LEU66 in the 10 individual trajectories. Tables with all entropy values and estimates of their statistical uncertainties. This Information is available free of charge via the Internet at http://pubs.acs.org 


\section{References}

(1) Sliwoski, G.; Kothiwale, S.; Meiler, J.; Lowe, E. W. Computational Methods in Drug Discovery. Pharmacol. Rev. 2014, 66, 334-395.

(2) Akke, M.; Brueschweiler, R.; Palmer, A. G. NMR order parameters and free energy: an analytical approach and its application to cooperative calcium $(2+)$ binding by calbindin D9k. J. Am. Chem. Soc. 1993, 115, 9832-9833.

(3) Marlow, M. S.; Dogan, J.; Frederick, K. K.; Valentine, K. G.; Wand, A. J. The role of conformational entropy in molecular recognition by calmodulin. Nat. Chem. Biol. 2010, 6, 352-358.

(4) Frederick, K. K.; Marlow, M. S.; Valentine, K. G.; Wand, A. J. Conformational Entropy in Molecular Recognition by Proteins. Nature 2007, 448, 325-329.

(5) McClendon, C. L.; Friedland, G.; Mobley, D. L.; Amirkhani, H.; Jacobson, M. P. Quantifying Correlations Between Allosteric Sites in Thermodynamic Ensembles. J. Chem. Theory Comput. 2009, 5, 2486-2502.

(6) Diehl, C.; Engström, O.; Delaine, T.; Håkansson, M.; Genheden, S.; Modig, K.; Leffler, H.; Ryde, U.; Nilsson, U. J.; Akke, M. Protein Flexibility and Conformational Entropy in Ligand Design Targeting the Carbohydrate Recognition Domain of Galectin-3. J. Am. Chem. Soc. 2010, 132, 14577-14589.

(7) Tzeng, S.-R.; Kalodimos, C. G. Protein activity regulation by conformational entropy. Nature 2012, 488, 236-240.

(8) Gill, M. L.; Byrd, R. A.; Palmer, A. G. Dynamics of GCN4 facilitate DNA interaction: a model-free analysis of an intrinsically disordered region. Phys. Chem. Chem. Phys. 2016, 18, 5839-5849. 
(9) MacRaild, C. A.; Daranas, A. H.; Bronowska, A.; Homans, S. W. Global Changes in Local Protein Dynamics Reduce the Entropic Cost of Carbohydrate Binding in the Arabinose-binding Protein. J. Mol. Biol. 2007, 368, 822-832.

(10) Syme, N. R.; Dennis, C.; Bronowska, A.; Paesen, G. C.; Homans, S. W. Comparison of Entropic Contributions to Binding in a Hydrophilic versus Hydrophobic Ligand-Protein Interaction. J. Am. Chem. Soc. 2010, 132, 8682-8689.

(11) Wallerstein, J.; Ekberg, V.; Ignjatović, M. M.; Kumar, R.; Caldararu, O.; Peterson, K.; Wernersson, S.; Brath, U.; Leffler, H.; Oksanen, E.; Logan, D. T.; Nilsson, U. J.; Ryde, U.; Akke, M. Entropy-Entropy Compensation between the Protein, Ligand, and Solvent Degrees of Freedom Fine-Tunes Affinity in Ligand Binding to Galectin-3C. JACS Au 2021, 1, 484-500.

(12) Päslack, C.; Das, C. K.; Schlitter, J.; Schäfer, L. V. Spectrally Resolved Estimation of Water Entropy in the Active Site of Human Carbonic Anhydrase II. J. Chem. Theory Comput. 2021, 17, 5409-5418.

(13) Juber, S.; Wingbermühle, S.; Nuernberger, P.; Clever, G. H.; Schäfer, L. V. Thermodynamic driving forces of guest confinement in a photoswitchable cage. Phys. Chem. Chem. Phys. 2021, 23, 7321-7332.

(14) Kasinath, V.; Sharp, K. A.; Wand, A. J. Microscopic Insights into the NMR RelaxationBased Protein Conformational Entropy Meter. J. Am. Chem. Soc. 2013, 135, 1509215100.

(15) Sharp, K. A.; O`Brien, E.; Kasinath, V.; Wand, A. J. On the relationship between NMR-derived amide order parameters and protein backbone entropy changes. Proteins 2015, 83, 922-930.

(16) Caro, J. A.; Harpole, K. W.; Kasinath, V.; Lim, J.; Granja, J.; Valentine, K. G.; 
Sharp, K. A.; Wand, A. J. Entropy in molecular recognition by proteins. Proc. Natl. Acad. Sci. U.S.A. 2017, 114, 6563-6568.

(17) Peng, J. W. Exposing the Moving Parts of Proteins with NMR Spectroscopy. J. Phys. Chem. Lett. 2012, 3, 1039-1051.

(18) Brainard, J. R.; Szabo, A. Theory for nuclear magnetic relaxation of probes in anisotropic systems: application of cholesterol in phospholipid vesicles. Biochemistry 1981, 20, 4618-28.

(19) Yang, D.; Kay, L. E. Contributions to Conformational Entropy Arising from Bond Vector Fluctuations Measured from NMR-Derived Order Parameters: Application to Protein Folding. J. Mol. Biol. 1996, 263, 369-382.

(20) Li, Z.; Raychaudhuri, S.; Wand, A. J. Insights into the local residual entropy of proteins provided by NMR relaxation. Prot. Sci. 1996, 5, 2647-2650.

(21) Yang, D.; Mok, Y. K.; Forman-Kay, J. D.; Farrow, N. A.; Kay, L. E. Contributions to protein entropy and heat capacity from bond vector motions measured by NMR spin relaxation. J. Mol. Biol. 1997, 272, 790-804.

(22) Spyracopoulos, L.; Sykes, B. D. Thermodynamic insights into proteins from NMR spin relaxation studies. Curr. Opin. Struct. Biol. 2001, 11, 555-559.

(23) Rajeshwar T, R.; Saharay, M.; Smith, J. C.; Krishnan, M. Correlated Response of Protein Side-Chain Fluctuations and Conformational Entropy to Ligand Binding. J. Phys. Chem. B 2021, XXX, XXX-XXX.

(24) Hoffmann, F.; Mulder, F. A. A.; Schäfer, L. V. Accurate Methyl Group Dynamics in Protein Simulations with AMBER Force Fields. J. Phys. Chem. B 2018, 122, 50385048 . 
(25) Hoffmann, F.; Xue, M.; Schäfer, L. V.; Mulder, F. A. A. Narrowing the gap between experimental and computational determination of methyl group dynamics in proteins. Phys. Chem. Chem. Phys. 2018, 20, 24577-24590.

(26) Hoffmann, F.; Mulder, F. A. A.; Schäfer, L. V. Predicting NMR relaxation of proteins from molecular dynamics simulations with accurate methyl rotation barriers. J. Chem. Phys. 2020, 152, 084102.

(27) Anderson, J. S.; Hernández, G.; LeMaster, D. M. ${ }^{13}$ C NMR Relaxation Analysis of Protein GB3 for the Assessment of Side Chain Dynamics Predictions by Current AMBER and CHARMM Force Fields. J. Chem. Theory Comput. 2020, 16, 2896-2913.

(28) Yang, D.; Mittermaier, A.; Mok, Y. K.; Kay, L. E. A Study of Protein Side-Chain Dynamics from New ${ }^{2} \mathrm{H}$ Auto-Correlation and ${ }^{13} \mathrm{C}$ Cross-correlation NMR Experiments: Application to the N-terminal SH3 Domain from Drk. J. Mol. Biol. 1998, 276, 939-954.

(29) Chou, J. J.; Case, D. A.; Bax, A. Insights into the Mobility of Methyl-Bearing Side Chains in Proteins from ${ }^{3} \mathrm{~J}_{C C}$ and ${ }^{3} \mathrm{~J}_{C N}$ couplings. J. Am. Chem. Soc. 2003, 125, $8959-8966$.

(30) Best, R. B.; Clarke, J.; Karplus, M. What Contributions to Protein Side-Chain Dynamics are Probed by NMR Experiments? A Molecular Dynamics Simulation Analysis. J. Mol. Biol. 2005, 349, 185-203.

(31) Salvi, N.; Abyzov, A.; Blackledge, M. Multi-Timescale Dynamics in Intrinsically Disordered Proteins from NMR Relaxation and Molecular Simulation. J. Phys. Chem. Lett. 2016, 7, 2483-2489.

(32) Salvi, N.; Abyzov, A.; Blackledge, M. Analytical Description of NMR Relaxation Highlights Correlated Dynamics in Intrinsically Disordered Proteins. Angew. Chem. Int. Ed. 2017, 56, 14020-14024. 
(33) Salvi, N.; Abyzov, A.; Blackledge, M. Solvent-dependent segmental dynamics in intrinsically disordered proteins. Sci. Adv. 2019, 5, eaax2348.

(34) Li, D.-W.; Brüschweiler, R. A Dictionary for Protein Side-Chain Entropies from NMR Order Parameters. J. Am. Chem. Soc. 2009, 131, 7226-7227.

(35) Li, D.-W.; Showalter, S. A.; Brüschweiler, R. Entropy Localization in Proteins. J. Phys. Chem. B 2010, 114, 16036-16044.

(36) Genheden, S.; Akke, M.; Ryde, U. Conformational Entropies and Order Parameters: Convergence, Reproducibility, and Transferability. J. Chem. Theory Comput. 2014, 10, $432-438$.

(37) Fleck, M.; Zagrovic, B. Configurational Entropy Components and Their Contribution to Biomolecular Complex Formation. J. Chem. Theory Comput. 2019, 15, 3844-3853.

(38) Best, R. B.; Hummer, G. Optimized Molecular Dynamics Force Fields Applied to the Helix-Coil Transition of Polypeptides. J. Phys. Chem. B 2009, 113, 9004-9015.

(39) Lindorff-Larsen, K.; Piana, S.; Palmo, K.; Maragakis, P.; Klepeis, J. L.; Dror, R. O.; Shaw, D. E. Improved Side-Chain Torsion Potentials for the Amber ff99SB Protein Force Field. Proteins 2010, 78, 1950-1958.

(40) Best, R. B.; Zhu, X.; Shim, J.; Lopes, P. E. M.; Mittal, J.; Feig, M.; MacKerell, A. D. Optimization of the Additive CHARMM All-Atom Protein Force Field Targeting Improved Sampling of the Backbone $\phi, \psi$ and Side-Chain $\chi_{1}$ and $\chi_{2}$ Dihedral Angles. $J$. Chem. Theory Comput. 2012, 8, 3257-3273.

(41) Abascal, J. L. F.; Vega, C. A General Purpose Model for the Condensed Phases of Water: TIP4P/2005. J. Chem. Phys. 2005, 123, 234505.

(42) Abraham, M. J.; Murtola, T.; Schulz, R.; Páll, S.; Smith, J. C.; Hess, B.; Lindahl, E. 
GROMACS: High Performance Molecular Simulations through Multi-Level Parallelism from Laptops to Supercomputers. SoftwareX 2015, 1-2, 19-25.

(43) Bussi, G.; Donadio, D.; Parrinello, M. Canonical Sampling Through Velocity Rescaling. J. Chem. Phys. 2007, 126, 014101.

(44) Miyamoto, S.; Kollman, P. A. Settle: An Analytical Version of the SHAKE and RATTLE Algorithm for Rigid Water Models. J. Comput. Chem. 1992, 13, 952-962.

(45) Hess, B. P-LINCS: A Parallel Linear Constraint Solver for Molecular Simulation. J. Chem. Theory Comput. 2008, 4, 116-122.

(46) Essmann, U.; Perera, L.; Berkowitz, M. L.; Darden, T.; Lee, H.; Pedersen, L. G. A Smooth Particle Mesh Ewald Method. J. Chem. Phys. 1995, 103, 8577-8593.

(47) Schlitter, J. Estimation of absolute and relative entropies of macromolecules using the covariance matrix. Chem. Phys. Lett. 1993, 215, 617-621.

(48) Lovell, S. C.; Word, J. M.; Richardson, J. S.; Richardson, D. C. The Penultimate Rotamer Library. Proteins 2000, 40, 389-408.

(49) Hensen, U.; Lange, O. F.; Grubmüller, H. Estimating Absolute Configurational Entropies of Macromolecules: The Minimally Coupled Subspace Approach. PLoS One 2010, 5, e9179.

(50) Fleck, M.; Polyansky, A. A.; Zagrovic, B. Self-Consistent Framework Connecting Experimental Proxies of Protein Dynamics with Configurational Entropy. J. Chem. Theory Comput. 2018, 14, 3796-3810.

(51) Wohlkönig, A.; Huet, J.; Looze, Y.; Wintjens, R. Structural relationships in the lysozyme superfamily: significant evidence for glycoside hydrolase signature motifs. PLoS One 2010, 5, e15388. 
(52) Vitalini, F.; Mey, A. S. J. S.; Noé, F.; Keller, B. G. Dynamic properties of force fields. J. Chem. Phys. 2015, 142, 084101.

(53) Kulkarni, M.; Söderhjelm, P. Free Energy Landscape and Rate Estimation of the Aromatic Ring Flips in Basic Pancreatic Trypsin Inhibitor Using Metadynamics. bioRxiv 2021,

(54) Best, R. B.; Clarke, J.; Karplus, M. The Origin of Protein Sidechain Order Parameter Distributions. J. Am. Chem. Soc. 2004, 126, 7734-7735.

(55) Hu, H.; Hermans, J.; Lee, A. L. Relating Side-Chain Mobility in Proteins to Rotameric Transitions: Insights from Molecular Dynamics Simulations and NMR. J. Biomol. NMR 2005, 32, 151-62.

(56) Allnér, O.; Foloppe, N.; Nilsson, L. Motions and Entropies in Proteins as Seen in NMR Relaxation Experiments and Molecular Dynamics Simulations. J. Phys. Chem. B 2015, 119, 1114-1128.

(57) Farès, C.; Lakomek, N.-A.; Walter, K. F. A.; Frank, B. T. C.; Meiler, J.; Becker, S.; Griesinger, C. Accessing ns- $-\mu$ s side chain dynamics in ubiquitin with methyl RDCs. J. Biomol. NMR 2009, 45, 23-44.

(58) Bowman, G. R. Accurately Modeling Nanosecond Protein Dynamics Requires at Least Microseconds of Simulation. J. Comput. Chem. 2016, 37, 558-566.

(59) Xiang, X.; Hansen, A. L.; Yu, L.; Jameson, G.; Bruschweiler-Li, L.; Yuan, C.; Brüschweiler, R. Observation of Sub-Microsecond Protein Methyl-Side Chain Dynamics by Nanoparticle-Assisted NMR Spin Relaxation. J. Am. Chem. Soc. 2021, XXX, $\mathrm{XXX}-\mathrm{XXX}$ 


\title{
Supporting Information:
}

\section{How much entropy is contained in NMR relaxation parameters?}

\author{
Falk Hoffmann, ${ }^{*, \dagger, \dagger}$ Frans A. A. Mulder, ${ }^{*, \Phi}$ and Lars V. Schäfer, ${ }^{*, \dagger}$ \\ $\dagger$ Center for Theoretical Chemistry, Ruhr University Bochum, D-44780 Bochum, Germany \\ $\ddagger$ Current address: Peptone Inc., Northcliff House, Young Street, London, England W8 5, \\ U.K. \\ \Interdisciplinary Nanoscience Center (iNANO) and Department of Chemistry, University \\ of Aarhus, DK-8000 Aarhus, Denmark
}

E-mail: falk@peptone.io; fmulder@chem.au.dk; lars.schaefer@ruhr-uni-bochum.de Phone: +49 (0)234 3221582 


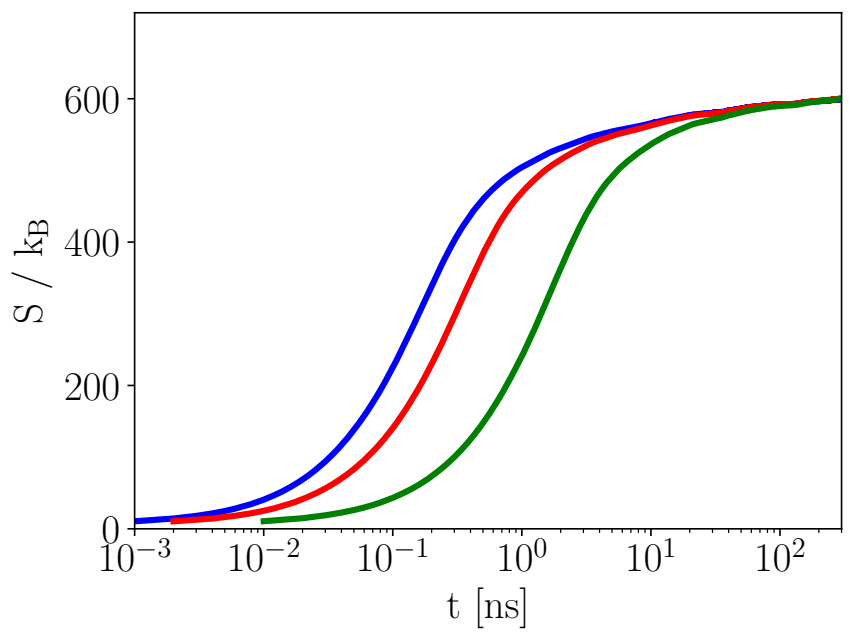

Figure S1: QHA backbone entropy build-up obtained from the AMBER ff99SB*-ILDN simulations with 1 ps (blue), 2 ps (red), and 10 ps (green) time spacing between the trajectory frames analyzed.

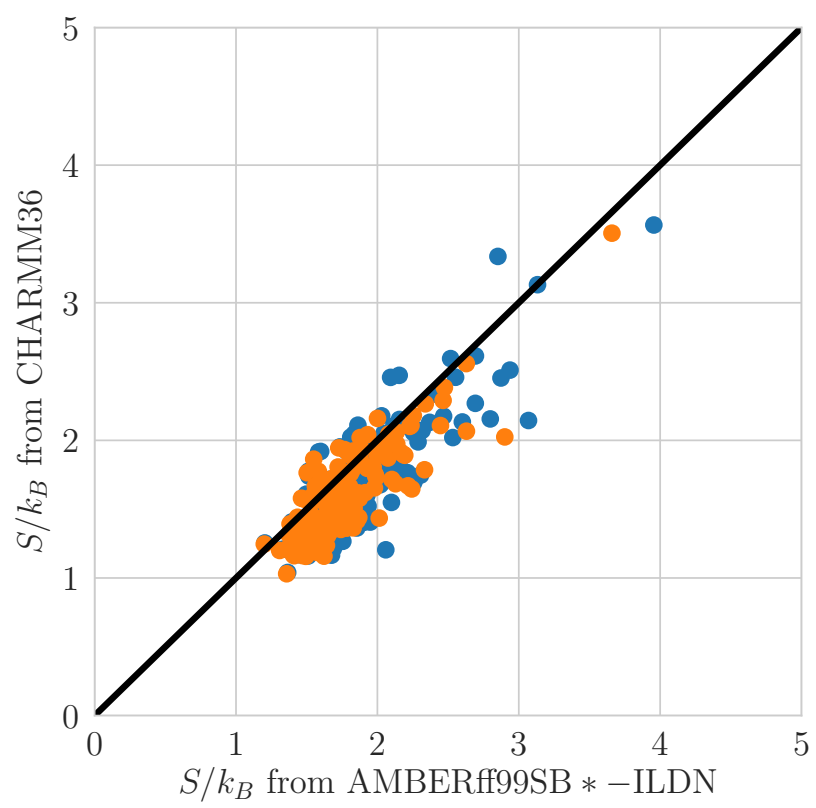

Figure S2: Correlation of the backbone entropy of all non-alanine residues at the tumbling time (orange) and in the long-time limit (blue) between the simulations with the AMBER ff99SB*-ILDN and the CHARMM36 force field. 

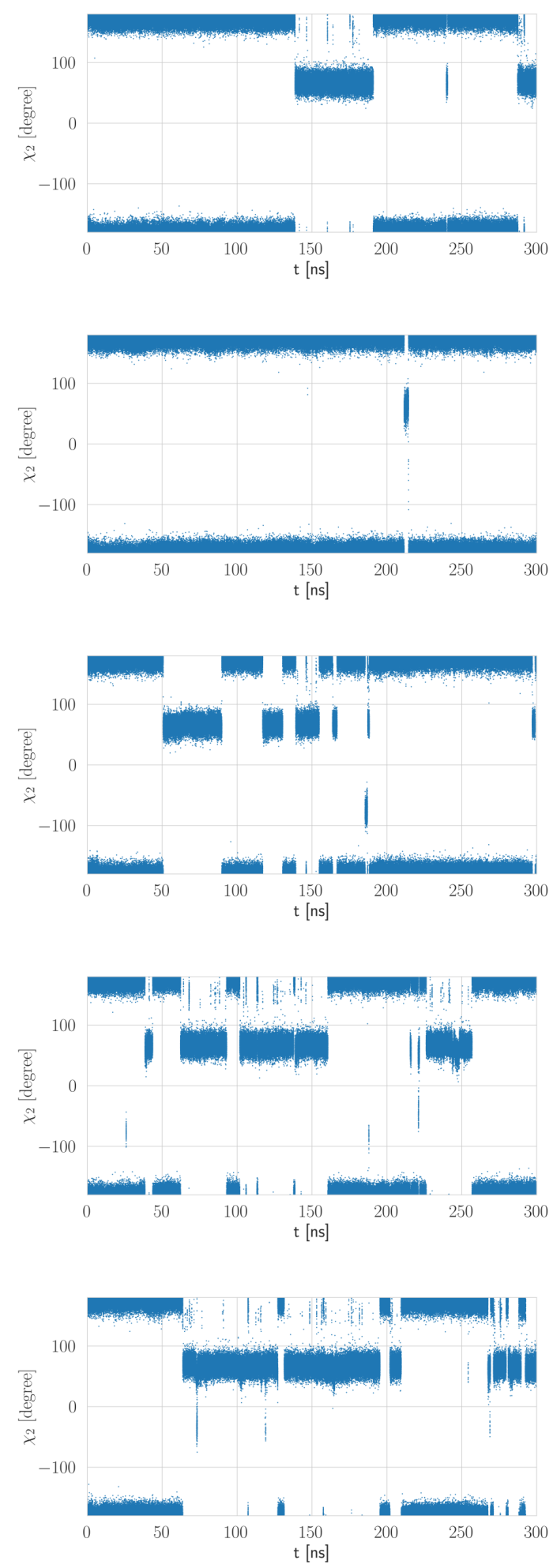
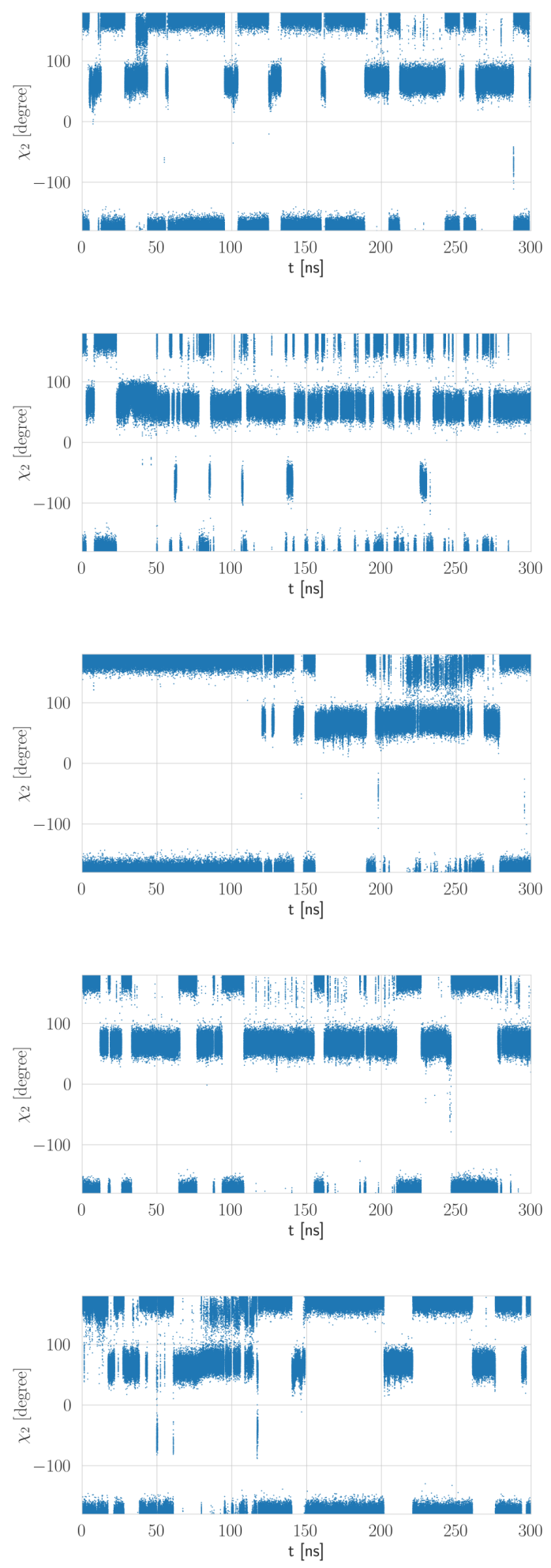

Figure S3: Side chain dihedral angle $\chi_{2}$ of LEU66 during the 10 individual simulations. 
Table S1: Backbone entropy $S / k_{\mathrm{B}}$ within the tumbling time $\tau_{\mathrm{R}}$ and in the long-time limit with the AMBER ff99SB*-ILDN force field. STD and SEM are the standard deviation and the standard error of the mean, respectively.

\begin{tabular}{|c|c|c|c|c|c|}
\hline Residue number & $S / k_{B}\left[\tau_{\mathbf{R}}\right]$ & $S / k_{B}\left[t_{\text {sim }}\right]$ & $S\left[\tau_{\mathbf{R}}\right] / S\left[t_{\operatorname{sim}}\right]$ & STD & SEM \\
\hline 2 & 1.7222 & 1.8622 & 0.925 & 0.0268 & 0.014 \\
\hline 3 & 1.3733 & 1.4190 & 0.968 & 0.0053 & 0.004 \\
\hline 4 & 1.5726 & 1.6902 & 0.930 & 0.0184 & 0.011 \\
\hline 5 & 1.7826 & 1.9060 & 0.935 & 0.0245 & 0.013 \\
\hline 6 & 1.4700 & 1.5225 & 0.966 & 0.0094 & 0.006 \\
\hline 7 & 1.4353 & 1.5145 & 0.948 & 0.0076 & 0.005 \\
\hline 8 & 1.6734 & 1.8366 & 0.911 & 0.0247 & 0.013 \\
\hline 9 & 1.8814 & 2.0461 & 0.920 & 0.0115 & 0.006 \\
\hline 10 & 1.9911 & 2.0935 & 0.951 & 0.0100 & 0.005 \\
\hline 11 & 2.2378 & 2.8524 & 0.785 & 0.0219 & 0.008 \\
\hline 12 & 2.2539 & 3.1326 & 0.719 & 0.0471 & 0.015 \\
\hline 13 & 2.4462 & 2.9379 & 0.833 & 0.0490 & 0.017 \\
\hline 14 & 2.3334 & 2.6007 & 0.897 & 0.0317 & 0.012 \\
\hline 15 & 2.1936 & 2.2877 & 0.959 & 0.0110 & 0.005 \\
\hline 16 & 2.0810 & 2.1558 & 0.965 & 0.0084 & 0.004 \\
\hline 17 & 1.5613 & 1.5904 & 0.982 & 0.0025 & 0.002 \\
\hline 18 & 1.9644 & 2.0495 & 0.958 & 0.0228 & 0.011 \\
\hline 19 & 2.1371 & 2.3698 & 0.902 & 0.0150 & 0.006 \\
\hline 20 & 2.0416 & 2.5180 & 0.811 & 0.0189 & 0.008 \\
\hline 21 & 1.8590 & 2.1543 & 0.863 & 0.0263 & 0.012 \\
\hline 22 & 2.1684 & 2.5335 & 0.856 & 0.0216 & 0.009 \\
\hline 23 & 2.4648 & 2.8739 & 0.858 & 0.0241 & 0.008 \\
\hline 24 & 2.2289 & 2.4683 & 0.903 & 0.0117 & 0.005 \\
\hline 25 & 1.6859 & 1.8868 & 0.894 & 0.0191 & 0.010 \\
\hline 26 & 1.5041 & 1.5730 & 0.956 & 0.0090 & 0.006 \\
\hline 27 & 1.5443 & 1.6084 & 0.960 & 0.0079 & 0.005 \\
\hline 28 & 1.6799 & 1.7797 & 0.944 & 0.0215 & 0.012 \\
\hline 29 & 2.1859 & 2.6926 & 0.812 & 0.0420 & 0.016 \\
\hline 30 & 1.9963 & 2.3162 & 0.862 & 0.0310 & 0.013 \\
\hline 31 & 1.8094 & 1.9418 & 0.932 & 0.0086 & 0.004 \\
\hline 32 & 1.8599 & 1.9176 & 0.970 & 0.0111 & 0.006 \\
\hline 33 & 1.9310 & 2.1167 & 0.912 & 0.0224 & 0.011 \\
\hline 34 & 1.4631 & 1.5545 & 0.941 & 0.0278 & 0.018 \\
\hline 35 & 1.8012 & 1.9088 & 0.944 & 0.0387 & 0.020 \\
\hline 36 & 1.8650 & 1.9254 & 0.969 & 0.0205 & 0.011 \\
\hline 38 & 2.4738 & 2.5531 & 0.969 & 0.0285 & 0.011 \\
\hline 39 & 1.3820 & 1.3951 & 0.991 & 0.0078 & 0.006 \\
\hline 40 & 1.5195 & 1.5292 & 0.994 & 0.0023 & 0.002 \\
\hline 41 & 1.5615 & 1.5869 & 0.984 & 0.0046 & 0.003 \\
\hline 42 & 1.5778 & 1.6013 & 0.985 & 0.0069 & 0.004 \\
\hline 43 & 1.5223 & 1.5424 & 0.987 & 0.0047 & 0.003 \\
\hline 44 & 1.6310 & 1.6656 & 0.979 & 0.0088 & 0.005 \\
\hline 45 & 1.5373 & 1.5849 & 0.970 & 0.0063 & 0.004 \\
\hline 46 & 1.4109 & 1.4411 & 0.979 & 0.0041 & 0.003 \\
\hline 47 & 1.4899 & 1.5130 & 0.985 & 0.0048 & 0.003 \\
\hline 48 & 1.8286 & 1.8548 & 0.986 & 0.0145 & 0.008 \\
\hline 49 & 1.8551 & 1.8737 & 0.990 & 0.0164 & 0.009 \\
\hline 50 & 1.9702 & 2.0718 & 0.951 & 0.0087 & 0.004 \\
\hline 51 & 2.0743 & 2.1250 & 0.976 & 0.0065 & 0.003 \\
\hline 52 & 1.8354 & 1.9338 & 0.949 & 0.0121 & 0.006 \\
\hline
\end{tabular}




\begin{tabular}{|c|c|c|c|c|c|}
\hline 53 & 1.9852 & 2.1008 & 0.945 & 0.0085 & 0.004 \\
\hline 54 & 2.0459 & 2.2550 & 0.907 & 0.0098 & 0.004 \\
\hline 55 & 1.5495 & 1.5843 & 0.978 & 0.0123 & 0.008 \\
\hline 56 & 1.8838 & 1.9191 & 0.982 & 0.0161 & 0.008 \\
\hline 57 & 1.7764 & 1.8822 & 0.944 & 0.0137 & 0.007 \\
\hline 58 & 1.8040 & 1.8627 & 0.968 & 0.0084 & 0.005 \\
\hline 59 & 1.8730 & 1.9254 & 0.973 & 0.0101 & 0.005 \\
\hline 60 & 1.5927 & 1.6327 & 0.976 & 0.0111 & 0.007 \\
\hline 61 & 1.7530 & 1.8187 & 0.964 & 0.0190 & 0.010 \\
\hline 62 & 1.6601 & 1.7112 & 0.970 & 0.0287 & 0.017 \\
\hline 63 & 1.4712 & 1.5008 & 0.980 & 0.0205 & 0.014 \\
\hline 64 & 1.5204 & 1.5938 & 0.954 & 0.0310 & 0.019 \\
\hline 65 & 1.6483 & 1.7240 & 0.956 & 0.0191 & 0.011 \\
\hline 66 & 1.6292 & 1.7557 & 0.928 & 0.0249 & 0.014 \\
\hline 67 & 1.5907 & 2.0594 & 0.772 & 0.0343 & 0.017 \\
\hline 68 & 1.6509 & 1.7519 & 0.942 & 0.0093 & 0.005 \\
\hline 69 & 1.7985 & 1.9498 & 0.922 & 0.0276 & 0.014 \\
\hline 70 & 1.5918 & 1.6401 & 0.971 & 0.0081 & 0.005 \\
\hline 71 & 1.5274 & 1.5803 & 0.967 & 0.0148 & 0.009 \\
\hline 72 & 1.5551 & 1.5851 & 0.981 & 0.0030 & 0.002 \\
\hline 73 & 1.4995 & 1.5299 & 0.980 & 0.0068 & 0.004 \\
\hline 74 & 1.4938 & 1.5107 & 0.989 & 0.0085 & 0.006 \\
\hline 75 & 1.4699 & 1.5024 & 0.978 & 0.0066 & 0.004 \\
\hline 76 & 1.5276 & 1.5503 & 0.985 & 0.0072 & 0.005 \\
\hline 77 & 1.7604 & 1.8115 & 0.972 & 0.0120 & 0.007 \\
\hline 78 & 1.6397 & 1.7099 & 0.959 & 0.0156 & 0.009 \\
\hline 79 & 1.9791 & 2.0206 & 0.979 & 0.0089 & 0.004 \\
\hline 80 & 2.6298 & 2.7984 & 0.940 & 0.0229 & 0.008 \\
\hline 81 & 2.1342 & 2.2480 & 0.949 & 0.0113 & 0.005 \\
\hline 82 & 1.7266 & 1.7348 & 0.995 & 0.0020 & 0.001 \\
\hline 83 & 2.0007 & 2.0302 & 0.985 & 0.0052 & 0.003 \\
\hline 84 & 1.8779 & 1.9094 & 0.984 & 0.0057 & 0.003 \\
\hline 85 & 1.4220 & 1.4461 & 0.983 & 0.0049 & 0.003 \\
\hline 87 & 1.6223 & 1.6756 & 0.968 & 0.0152 & 0.009 \\
\hline 88 & 1.5377 & 1.5787 & 0.974 & 0.0052 & 0.003 \\
\hline 89 & 1.8686 & 1.9120 & 0.977 & 0.0066 & 0.003 \\
\hline 90 & 2.2431 & 2.3060 & 0.973 & 0.0151 & 0.007 \\
\hline 91 & 2.1287 & 2.2129 & 0.962 & 0.0155 & 0.007 \\
\hline 92 & 1.7420 & 1.7712 & 0.984 & 0.0109 & 0.006 \\
\hline 93 & 1.8785 & 1.8931 & 0.992 & 0.0104 & 0.006 \\
\hline 94 & 1.7132 & 1.7240 & 0.994 & 0.0021 & 0.001 \\
\hline 95 & 1.1998 & 1.2037 & 0.997 & 0.0013 & 0.001 \\
\hline 96 & 1.4104 & 1.4165 & 0.996 & 0.0023 & 0.002 \\
\hline 97 & 1.4347 & 1.4375 & 0.998 & 0.0013 & 0.001 \\
\hline 98 & 1.4679 & 1.4755 & 0.995 & 0.0017 & 0.001 \\
\hline 99 & 1.3979 & 1.4046 & 0.995 & 0.0033 & 0.002 \\
\hline 100 & 1.4050 & 1.4182 & 0.991 & 0.0017 & 0.001 \\
\hline 101 & 1.4435 & 1.4634 & 0.986 & 0.0057 & 0.004 \\
\hline 102 & 1.4024 & 1.4294 & 0.981 & 0.0023 & 0.002 \\
\hline 103 & 1.3088 & 1.3313 & 0.983 & 0.0091 & 0.007 \\
\hline 104 & 1.7418 & 1.7929 & 0.972 & 0.0167 & 0.009 \\
\hline 105 & 2.1022 & 2.1518 & 0.977 & 0.0187 & 0.009 \\
\hline 106 & 2.0314 & 2.2001 & 0.923 & 0.0141 & 0.006 \\
\hline 107 & 1.7671 & 1.8000 & 0.982 & 0.0065 & 0.004 \\
\hline 108 & 1.7151 & 1.7830 & 0.962 & 0.0095 & 0.005 \\
\hline 109 & 1.6246 & 1.6647 & 0.976 & 0.0117 & 0.007 \\
\hline
\end{tabular}




\begin{tabular}{|c|c|c|c|c|c|}
\hline 110 & 1.9136 & 1.9477 & 0.982 & 0.0114 & 0.006 \\
\hline 111 & 1.7129 & 1.7910 & 0.956 & 0.0157 & 0.009 \\
\hline 112 & 1.5829 & 1.5988 & 0.990 & 0.0039 & 0.002 \\
\hline 113 & 1.8019 & 1.8115 & 0.995 & 0.0030 & 0.002 \\
\hline 114 & 1.8190 & 1.8672 & 0.974 & 0.0112 & 0.006 \\
\hline 115 & 1.7909 & 1.8285 & 0.979 & 0.0089 & 0.005 \\
\hline 116 & 1.6675 & 1.7133 & 0.973 & 0.0106 & 0.006 \\
\hline 117 & 1.5960 & 1.6371 & 0.975 & 0.0072 & 0.004 \\
\hline 118 & 1.4668 & 1.4728 & 0.996 & 0.0020 & 0.001 \\
\hline 119 & 1.5094 & 1.5212 & 0.992 & 0.0031 & 0.002 \\
\hline 120 & 1.4791 & 1.4879 & 0.994 & 0.0019 & 0.001 \\
\hline 121 & 1.4549 & 1.4610 & 0.996 & 0.0021 & 0.001 \\
\hline 122 & 1.6008 & 1.6159 & 0.991 & 0.0046 & 0.003 \\
\hline 123 & 1.6468 & 1.6668 & 0.988 & 0.0064 & 0.004 \\
\hline 124 & 1.5032 & 1.5129 & 0.994 & 0.0043 & 0.003 \\
\hline 125 & 1.7572 & 1.7904 & 0.982 & 0.0132 & 0.007 \\
\hline 126 & 1.6962 & 1.7329 & 0.979 & 0.0089 & 0.005 \\
\hline 127 & 1.8162 & 1.8611 & 0.976 & 0.0091 & 0.005 \\
\hline 128 & 1.8177 & 1.8492 & 0.983 & 0.0066 & 0.004 \\
\hline 129 & 1.5443 & 1.6137 & 0.957 & 0.0086 & 0.005 \\
\hline 130 & 1.7274 & 1.7519 & 0.986 & 0.0047 & 0.003 \\
\hline 131 & 2.0129 & 2.0993 & 0.959 & 0.0092 & 0.004 \\
\hline 132 & 1.7125 & 1.7670 & 0.969 & 0.0101 & 0.006 \\
\hline 133 & 1.5462 & 1.6179 & 0.956 & 0.0073 & 0.005 \\
\hline 134 & 1.7680 & 1.7833 & 0.991 & 0.0045 & 0.003 \\
\hline 135 & 2.6287 & 2.6935 & 0.976 & 0.0154 & 0.006 \\
\hline 136 & 2.3388 & 2.3961 & 0.976 & 0.0127 & 0.005 \\
\hline 137 & 1.5151 & 1.5351 & 0.987 & 0.0061 & 0.004 \\
\hline 138 & 1.4790 & 1.4908 & 0.992 & 0.0018 & 0.001 \\
\hline 139 & 1.6347 & 1.6470 & 0.993 & 0.0026 & 0.002 \\
\hline 140 & 2.2163 & 2.2577 & 0.982 & 0.0051 & 0.002 \\
\hline 141 & 1.9969 & 2.0120 & 0.992 & 0.0070 & 0.003 \\
\hline 142 & 1.4814 & 1.4973 & 0.989 & 0.0031 & 0.002 \\
\hline 144 & 1.6188 & 1.6455 & 0.984 & 0.0033 & 0.002 \\
\hline 145 & 1.4252 & 1.4595 & 0.977 & 0.0037 & 0.003 \\
\hline 146 & 1.4462 & 1.4594 & 0.991 & 0.0015 & 0.001 \\
\hline 147 & 1.4972 & 1.5127 & 0.990 & 0.0015 & 0.001 \\
\hline 148 & 1.4030 & 1.5185 & 0.924 & 0.0057 & 0.004 \\
\hline 149 & 1.3583 & 1.3654 & 0.995 & 0.0045 & 0.003 \\
\hline 150 & 1.3767 & 1.3829 & 0.995 & 0.0012 & 0.001 \\
\hline 151 & 1.4521 & 1.4711 & 0.987 & 0.0024 & 0.002 \\
\hline 152 & 1.4549 & 1.4587 & 0.997 & 0.0013 & 0.001 \\
\hline 153 & 1.4321 & 1.4399 & 0.995 & 0.0033 & 0.002 \\
\hline 154 & 1.7167 & 1.7289 & 0.993 & 0.0043 & 0.003 \\
\hline 155 & 1.8281 & 1.8404 & 0.993 & 0.0030 & 0.002 \\
\hline 156 & 1.6109 & 1.6192 & 0.995 & 0.0027 & 0.002 \\
\hline 157 & 1.8216 & 1.8479 & 0.986 & 0.0024 & 0.001 \\
\hline 158 & 1.7890 & 1.8807 & 0.951 & 0.0070 & 0.004 \\
\hline 159 & 1.8413 & 1.8883 & 0.975 & 0.0084 & 0.004 \\
\hline 160 & 1.9287 & 1.9827 & 0.973 & 0.0165 & 0.008 \\
\hline 161 & 2.9010 & 3.0689 & 0.945 & 0.0284 & 0.009 \\
\hline 162 & 3.6589 & 3.9553 & 0.925 & 0.0195 & 0.005 \\
\hline
\end{tabular}


Table S2: Backbone entropy $S / k_{\mathrm{B}}$ within the tumbling time $\tau_{\mathrm{R}}$ and in the long-time limit with the CHARMM36 force field. STD and SEM are the standard deviation and the standard error of the mean, respectively.

\begin{tabular}{|c|c|c|c|c|c|}
\hline Residue number & $S / k_{B}\left[\tau_{\mathbf{R}}\right]$ & $S / k_{B}\left[t_{\operatorname{sim}}\right]$ & $S\left[\tau_{\mathbf{R}}\right] / S\left[t_{\operatorname{sim}}\right]$ & STD & SEM \\
\hline 2 & 1.8065 & 2.1104 & 0.856 & 0.0562 & 0.027 \\
\hline 3 & 1.3168 & 1.3597 & 0.968 & 0.0152 & 0.011 \\
\hline 4 & 1.1863 & 1.2160 & 0.976 & 0.0269 & 0.022 \\
\hline 5 & 1.3621 & 1.4048 & 0.970 & 0.0241 & 0.017 \\
\hline 6 & 1.2736 & 1.4178 & 0.898 & 0.0537 & 0.038 \\
\hline 7 & 1.4413 & 1.7408 & 0.828 & 0.0630 & 0.036 \\
\hline 8 & 1.5677 & 2.0471 & 0.766 & 0.0575 & 0.028 \\
\hline 9 & 1.6117 & 2.0502 & 0.786 & 0.0422 & 0.021 \\
\hline 10 & 1.7306 & 2.4578 & 0.704 & 0.0827 & 0.034 \\
\hline 11 & 2.1088 & 3.3367 & 0.632 & 0.0587 & 0.018 \\
\hline 12 & 2.1805 & 3.1312 & 0.696 & 0.0488 & 0.016 \\
\hline 13 & 2.1077 & 2.5106 & 0.840 & 0.0399 & 0.016 \\
\hline 14 & 1.7864 & 2.1338 & 0.837 & 0.0285 & 0.013 \\
\hline 15 & 1.8917 & 1.9890 & 0.951 & 0.0196 & 0.010 \\
\hline 16 & 2.0455 & 2.1519 & 0.951 & 0.0140 & 0.006 \\
\hline 17 & 1.6676 & 1.7210 & 0.969 & 0.0110 & 0.006 \\
\hline 18 & 1.8090 & 1.9742 & 0.916 & 0.0478 & 0.024 \\
\hline 19 & 1.9725 & 2.1309 & 0.926 & 0.0239 & 0.011 \\
\hline 20 & 1.9678 & 2.5942 & 0.759 & 0.0391 & 0.015 \\
\hline 21 & 1.8831 & 2.4726 & 0.762 & 0.0311 & 0.013 \\
\hline 22 & 1.9108 & 2.0204 & 0.946 & 0.0359 & 0.018 \\
\hline 23 & 2.2905 & 2.4532 & 0.934 & 0.0159 & 0.006 \\
\hline 24 & 2.1015 & 2.1752 & 0.966 & 0.0312 & 0.014 \\
\hline 25 & 1.6292 & 1.7543 & 0.929 & 0.0509 & 0.029 \\
\hline 26 & 1.4288 & 1.5278 & 0.935 & 0.0584 & 0.038 \\
\hline 27 & 1.4400 & 1.5056 & 0.956 & 0.0087 & 0.006 \\
\hline 28 & 1.7230 & 1.9242 & 0.895 & 0.0131 & 0.007 \\
\hline 29 & 1.8964 & 2.2691 & 0.836 & 0.0455 & 0.020 \\
\hline 30 & 1.9130 & 2.0726 & 0.923 & 0.0369 & 0.018 \\
\hline 31 & 1.7600 & 1.8569 & 0.948 & 0.0513 & 0.028 \\
\hline 32 & 1.9230 & 1.9738 & 0.974 & 0.0320 & 0.016 \\
\hline 33 & 2.0413 & 2.1054 & 0.970 & 0.0222 & 0.011 \\
\hline 34 & 1.5795 & 1.6014 & 0.986 & 0.0128 & 0.008 \\
\hline 35 & 1.6196 & 1.6316 & 0.993 & 0.0050 & 0.003 \\
\hline 36 & 1.6183 & 1.6726 & 0.968 & 0.0075 & 0.004 \\
\hline 38 & 2.3838 & 2.4585 & 0.970 & 0.0068 & 0.003 \\
\hline 39 & 1.3934 & 1.4078 & 0.990 & 0.0036 & 0.003 \\
\hline 40 & 1.3764 & 1.3798 & 0.998 & 0.0014 & 0.001 \\
\hline 41 & 1.2317 & 1.2331 & 0.999 & 0.0031 & 0.002 \\
\hline 42 & 1.2339 & 1.2371 & 0.997 & 0.0019 & 0.002 \\
\hline 43 & 1.3030 & 1.3116 & 0.993 & 0.0041 & 0.003 \\
\hline 44 & 1.2701 & 1.2870 & 0.987 & 0.0039 & 0.003 \\
\hline 45 & 1.3596 & 1.3704 & 0.992 & 0.0017 & 0.001 \\
\hline 46 & 1.1617 & 1.1693 & 0.993 & 0.0059 & 0.005 \\
\hline 47 & 1.3242 & 1.3266 & 0.998 & 0.0018 & 0.001 \\
\hline 48 & 1.3642 & 1.3633 & 1.001 & 0.0044 & 0.003 \\
\hline 49 & 1.4156 & 1.4171 & 0.999 & 0.0032 & 0.002 \\
\hline 50 & 1.8066 & 1.8318 & 0.986 & 0.0053 & 0.003 \\
\hline 51 & 1.8718 & 1.8942 & 0.988 & 0.0035 & 0.002 \\
\hline 52 & 1.5072 & 1.5225 & 0.990 & 0.0068 & 0.004 \\
\hline
\end{tabular}




\begin{tabular}{|c|c|c|c|c|c|}
\hline 53 & 1.7505 & 1.7726 & 0.988 & 0.0053 & 0.003 \\
\hline 54 & 1.9065 & 2.0567 & 0.927 & 0.0048 & 0.002 \\
\hline 55 & 1.8632 & 1.9159 & 0.973 & 0.0060 & 0.003 \\
\hline 56 & 1.9404 & 1.9692 & 0.985 & 0.0042 & 0.002 \\
\hline 57 & 1.5894 & 1.6883 & 0.941 & 0.0147 & 0.009 \\
\hline 58 & 1.6640 & 1.6969 & 0.981 & 0.0100 & 0.006 \\
\hline 59 & 1.5787 & 1.5989 & 0.987 & 0.0108 & 0.007 \\
\hline 60 & 1.3840 & 1.4073 & 0.983 & 0.0055 & 0.004 \\
\hline 61 & 1.3910 & 1.4119 & 0.985 & 0.0024 & 0.002 \\
\hline 62 & 1.3305 & 1.3517 & 0.984 & 0.0081 & 0.006 \\
\hline 63 & 1.3063 & 1.3227 & 0.988 & 0.0083 & 0.006 \\
\hline 64 & 1.3371 & 1.3754 & 0.972 & 0.0079 & 0.006 \\
\hline 65 & 1.3075 & 1.3194 & 0.991 & 0.0041 & 0.003 \\
\hline 66 & 1.2556 & 1.2666 & 0.991 & 0.0078 & 0.006 \\
\hline 67 & 1.1944 & 1.2048 & 0.991 & 0.0127 & 0.011 \\
\hline 68 & 1.3614 & 1.3745 & 0.990 & 0.0030 & 0.002 \\
\hline 69 & 1.3953 & 1.4062 & 0.992 & 0.0024 & 0.002 \\
\hline 70 & 1.2973 & 1.3072 & 0.992 & 0.0025 & 0.002 \\
\hline 71 & 1.2214 & 1.2253 & 0.997 & 0.0024 & 0.002 \\
\hline 72 & 1.2210 & 1.2229 & 0.998 & 0.0055 & 0.005 \\
\hline 73 & 1.3952 & 1.4007 & 0.996 & 0.0019 & 0.001 \\
\hline 74 & 1.1573 & 1.1599 & 0.998 & 0.0030 & 0.003 \\
\hline 75 & 1.1627 & 1.1708 & 0.993 & 0.0034 & 0.003 \\
\hline 76 & 1.4689 & 1.4833 & 0.990 & 0.0071 & 0.005 \\
\hline 77 & 1.4717 & 1.4845 & 0.991 & 0.0099 & 0.007 \\
\hline 78 & 1.2368 & 1.2517 & 0.988 & 0.0091 & 0.007 \\
\hline 79 & 1.6549 & 1.6776 & 0.986 & 0.0085 & 0.005 \\
\hline 80 & 2.0667 & 2.1558 & 0.959 & 0.0141 & 0.007 \\
\hline 81 & 2.0619 & 2.0973 & 0.983 & 0.0029 & 0.001 \\
\hline 82 & 1.9456 & 1.9525 & 0.996 & 0.0021 & 0.001 \\
\hline 83 & 2.1599 & 2.1783 & 0.992 & 0.0052 & 0.002 \\
\hline 84 & 2.0204 & 2.0416 & 0.990 & 0.0032 & 0.002 \\
\hline 85 & 1.2086 & 1.2131 & 0.996 & 0.0031 & 0.003 \\
\hline 87 & 1.1584 & 1.1657 & 0.994 & 0.0056 & 0.005 \\
\hline 88 & 1.2774 & 1.2877 & 0.992 & 0.0072 & 0.006 \\
\hline 89 & 1.4398 & 1.4732 & 0.977 & 0.0180 & 0.012 \\
\hline 90 & 1.6469 & 1.7476 & 0.942 & 0.0298 & 0.017 \\
\hline 91 & 1.6845 & 1.7653 & 0.954 & 0.0221 & 0.013 \\
\hline 92 & 1.6783 & 1.7283 & 0.971 & 0.0114 & 0.007 \\
\hline 93 & 1.8626 & 1.9083 & 0.976 & 0.0229 & 0.012 \\
\hline 94 & 1.5793 & 1.6010 & 0.986 & 0.0081 & 0.005 \\
\hline 95 & 1.2464 & 1.2545 & 0.994 & 0.0046 & 0.004 \\
\hline 96 & 1.2667 & 1.2699 & 0.997 & 0.0032 & 0.003 \\
\hline 97 & 1.3487 & 1.3532 & 0.997 & 0.0013 & 0.001 \\
\hline 98 & 1.3576 & 1.3642 & 0.995 & 0.0013 & 0.001 \\
\hline 99 & 1.2564 & 1.2605 & 0.997 & 0.0019 & 0.002 \\
\hline 100 & 1.3075 & 1.3163 & 0.993 & 0.0068 & 0.005 \\
\hline 101 & 1.2501 & 1.2643 & 0.989 & 0.0104 & 0.008 \\
\hline 102 & 1.1725 & 1.1870 & 0.988 & 0.0073 & 0.006 \\
\hline 103 & 1.1999 & 1.2091 & 0.992 & 0.0034 & 0.003 \\
\hline 104 & 1.3529 & 1.3657 & 0.991 & 0.0101 & 0.007 \\
\hline 105 & 1.7160 & 1.8302 & 0.938 & 0.0245 & 0.013 \\
\hline 106 & 1.9203 & 2.1057 & 0.912 & 0.0129 & 0.006 \\
\hline 107 & 1.6556 & 1.7967 & 0.921 & 0.0080 & 0.004 \\
\hline 108 & 1.4255 & 1.4863 & 0.959 & 0.0086 & 0.006 \\
\hline 109 & 1.6905 & 1.7170 & 0.985 & 0.0099 & 0.006 \\
\hline
\end{tabular}




\begin{tabular}{|c|c|c|c|c|c|}
\hline 110 & 1.6200 & 1.6686 & 0.971 & 0.0140 & 0.008 \\
\hline 111 & 1.5356 & 1.6910 & 0.908 & 0.0240 & 0.014 \\
\hline 112 & 1.7748 & 1.9197 & 0.925 & 0.0159 & 0.008 \\
\hline 113 & 1.8934 & 2.0234 & 0.936 & 0.0209 & 0.010 \\
\hline 114 & 1.7805 & 1.9563 & 0.910 & 0.0236 & 0.012 \\
\hline 115 & 1.5026 & 1.5411 & 0.975 & 0.0081 & 0.005 \\
\hline 116 & 1.3805 & 1.3932 & 0.991 & 0.0071 & 0.005 \\
\hline 117 & 1.3603 & 1.3797 & 0.986 & 0.0075 & 0.005 \\
\hline 118 & 1.3442 & 1.3484 & 0.997 & 0.0018 & 0.001 \\
\hline 119 & 1.3234 & 1.3419 & 0.986 & 0.0063 & 0.005 \\
\hline 120 & 1.3642 & 1.3798 & 0.989 & 0.0019 & 0.001 \\
\hline 121 & 1.3542 & 1.3577 & 0.997 & 0.0023 & 0.002 \\
\hline 122 & 1.5646 & 1.5852 & 0.987 & 0.0090 & 0.006 \\
\hline 123 & 1.5942 & 1.6251 & 0.981 & 0.0102 & 0.006 \\
\hline 124 & 1.7649 & 1.7757 & 0.994 & 0.0027 & 0.002 \\
\hline 125 & 1.7238 & 1.7870 & 0.965 & 0.0277 & 0.015 \\
\hline 126 & 1.5318 & 1.5558 & 0.985 & 0.0202 & 0.013 \\
\hline 127 & 1.4148 & 1.4380 & 0.984 & 0.0032 & 0.002 \\
\hline 128 & 1.4667 & 1.4995 & 0.978 & 0.0046 & 0.003 \\
\hline 129 & 1.3298 & 1.3436 & 0.990 & 0.0059 & 0.004 \\
\hline 130 & 1.3904 & 1.4140 & 0.983 & 0.0066 & 0.005 \\
\hline 131 & 1.4352 & 1.5491 & 0.926 & 0.0101 & 0.007 \\
\hline 132 & 1.5374 & 1.6026 & 0.959 & 0.0186 & 0.012 \\
\hline 133 & 1.5999 & 1.6666 & 0.960 & 0.0176 & 0.011 \\
\hline 134 & 1.9329 & 1.9570 & 0.988 & 0.0054 & 0.003 \\
\hline 135 & 2.5579 & 2.6135 & 0.979 & 0.0190 & 0.007 \\
\hline 136 & 2.2655 & 2.3360 & 0.970 & 0.0237 & 0.010 \\
\hline 137 & 1.4127 & 1.4388 & 0.982 & 0.0036 & 0.003 \\
\hline 138 & 1.2857 & 1.2939 & 0.994 & 0.0037 & 0.003 \\
\hline 139 & 1.4584 & 1.4771 & 0.987 & 0.0127 & 0.009 \\
\hline 140 & 1.6698 & 1.6944 & 0.985 & 0.0180 & 0.011 \\
\hline 141 & 1.8977 & 1.9545 & 0.971 & 0.0107 & 0.005 \\
\hline 142 & 1.5728 & 1.6092 & 0.977 & 0.0183 & 0.011 \\
\hline 144 & 1.3855 & 1.4056 & 0.986 & 0.0129 & 0.009 \\
\hline 145 & 1.2770 & 1.3474 & 0.948 & 0.0213 & 0.016 \\
\hline 146 & 1.2902 & 1.3322 & 0.968 & 0.0104 & 0.008 \\
\hline 147 & 1.3566 & 1.3839 & 0.980 & 0.0048 & 0.003 \\
\hline 148 & 1.2370 & 1.2770 & 0.969 & 0.0140 & 0.011 \\
\hline 149 & 1.0306 & 1.0401 & 0.991 & 0.0058 & 0.006 \\
\hline 150 & 1.2263 & 1.2317 & 0.996 & 0.0031 & 0.003 \\
\hline 151 & 1.3487 & 1.3655 & 0.988 & 0.0028 & 0.002 \\
\hline 152 & 1.3061 & 1.3150 & 0.993 & 0.0039 & 0.003 \\
\hline 153 & 1.3549 & 1.3671 & 0.991 & 0.0044 & 0.003 \\
\hline 154 & 1.5002 & 1.5255 & 0.983 & 0.0083 & 0.005 \\
\hline 155 & 1.9227 & 1.9667 & 0.978 & 0.0141 & 0.007 \\
\hline 156 & 1.5660 & 1.5840 & 0.989 & 0.0136 & 0.009 \\
\hline 157 & 1.4635 & 1.7554 & 0.834 & 0.0264 & 0.015 \\
\hline 158 & 1.5202 & 1.6456 & 0.924 & 0.0313 & 0.019 \\
\hline 159 & 1.8755 & 1.9276 & 0.973 & 0.0156 & 0.008 \\
\hline 160 & 1.7967 & 1.8402 & 0.976 & 0.0139 & 0.008 \\
\hline 161 & 2.0250 & 2.1444 & 0.944 & 0.0526 & 0.025 \\
\hline 162 & 3.5057 & 3.5650 & 0.983 & 0.0225 & 0.006 \\
\hline
\end{tabular}


Table S3: Side chain entropy $S /\left(k_{\mathrm{B}} N_{\chi}\right)$ within the tumbling time $\tau_{\mathrm{R}}$ and in the long-time limit with the AMBER ff99SB*-ILDN force field. STD and SEM are the standard deviation and the standard error of the mean, respectively.

\begin{tabular}{|c|c|c|c|c|c|}
\hline Residue number & $S /\left(k_{B} N_{\chi}\right)\left[\tau_{\mathbf{R}}\right]$ & $S /\left(k_{B} N_{\chi}\right)\left[t_{\text {sim }}\right]$ & $S\left[\tau_{\mathbf{R}}\right] / S\left[t_{\operatorname{sim}}\right]$ & STD & SEM \\
\hline 1 & 0.3133 & 0.3620 & 0.865 & 0.0091 & 0.025 \\
\hline 2 & 0.2820 & 0.4850 & 0.581 & 0.0133 & 0.027 \\
\hline 3 & 0.1147 & 0.2545 & 0.451 & 0.0222 & 0.087 \\
\hline 4 & 0.2069 & 0.8371 & 0.247 & 0.0439 & 0.052 \\
\hline 5 & 0.4097 & 0.5102 & 0.803 & 0.0125 & 0.025 \\
\hline 6 & 0.1424 & 0.1790 & 0.796 & 0.0174 & 0.097 \\
\hline 7 & 0.1978 & 0.3471 & 0.570 & 0.0397 & 0.114 \\
\hline 8 & 0.3157 & 0.6811 & 0.463 & 0.0170 & 0.025 \\
\hline 9 & 0.3086 & 0.5239 & 0.589 & 0.0089 & 0.017 \\
\hline 10 & 0.0445 & 0.2547 & 0.175 & 0.0052 & 0.020 \\
\hline 11 & 0.3882 & 0.6431 & 0.604 & 0.0192 & 0.030 \\
\hline 13 & 0.1765 & 0.2822 & 0.626 & 0.0235 & 0.083 \\
\hline 14 & 0.1650 & 0.3534 & 0.467 & 0.0188 & 0.053 \\
\hline 15 & 0.0887 & 0.1587 & 0.559 & 0.0191 & 0.121 \\
\hline 16 & 0.4947 & 0.5955 & 0.831 & 0.0057 & 0.010 \\
\hline 17 & 0.0370 & 0.1332 & 0.278 & 0.0076 & 0.057 \\
\hline 18 & 0.0001 & 0.0002 & 0.802 & 0.0000 & 0.210 \\
\hline 19 & 0.3959 & 0.4960 & 0.798 & 0.0074 & 0.015 \\
\hline 20 & 0.4654 & 0.6920 & 0.673 & 0.0089 & 0.013 \\
\hline 21 & 0.5961 & 0.6992 & 0.853 & 0.0134 & 0.019 \\
\hline 22 & 0.5965 & 0.7620 & 0.783 & 0.0113 & 0.015 \\
\hline 24 & 0.0869 & 0.4499 & 0.193 & 0.0189 & 0.042 \\
\hline 25 & 0.0016 & 0.0021 & 0.754 & 0.0005 & 0.257 \\
\hline 26 & 0.3279 & 0.7361 & 0.445 & 0.0230 & 0.031 \\
\hline 27 & 0.2687 & 0.3464 & 0.776 & 0.0148 & 0.043 \\
\hline 29 & 0.1706 & 0.3186 & 0.536 & 0.0195 & 0.061 \\
\hline 31 & 0.0418 & 0.0615 & 0.679 & 0.0060 & 0.098 \\
\hline 32 & 0.3315 & 0.5142 & 0.645 & 0.0102 & 0.020 \\
\hline 33 & 0.0125 & 0.0532 & 0.235 & 0.0039 & 0.073 \\
\hline 34 & 0.0409 & 0.3379 & 0.121 & 0.0149 & 0.044 \\
\hline 35 & 0.3173 & 0.4047 & 0.784 & 0.0133 & 0.033 \\
\hline 36 & 0.8637 & 0.9716 & 0.889 & 0.0105 & 0.011 \\
\hline 37 & 0.3201 & 0.3282 & 0.976 & 0.0044 & 0.014 \\
\hline 38 & 0.4562 & 0.5090 & 0.896 & 0.0140 & 0.028 \\
\hline 39 & 0.0802 & 0.1051 & 0.762 & 0.0055 & 0.053 \\
\hline 40 & 0.7217 & 0.8037 & 0.898 & 0.0114 & 0.014 \\
\hline 43 & 0.2795 & 0.3402 & 0.821 & 0.0078 & 0.023 \\
\hline 44 & 0.3717 & 0.4093 & 0.908 & 0.0091 & 0.022 \\
\hline 45 & 0.3788 & 0.5397 & 0.702 & 0.0074 & 0.014 \\
\hline 46 & 0.0075 & 0.0213 & 0.355 & 0.0049 & 0.229 \\
\hline 47 & 0.2035 & 0.5057 & 0.402 & 0.0229 & 0.045 \\
\hline 48 & 0.5587 & 0.6238 & 0.896 & 0.0039 & 0.006 \\
\hline 50 & 0.0544 & 0.2147 & 0.253 & 0.0105 & 0.049 \\
\hline 52 & 0.3123 & 0.5462 & 0.572 & 0.0187 & 0.034 \\
\hline 53 & 0.5849 & 0.8466 & 0.691 & 0.0142 & 0.017 \\
\hline 54 & 0.0212 & 0.0441 & 0.481 & 0.0079 & 0.180 \\
\hline 55 & 0.5940 & 0.7574 & 0.784 & 0.0216 & 0.029 \\
\hline 57 & 0.6333 & 1.0979 & 0.577 & 0.0223 & 0.020 \\
\hline 58 & 0.0238 & 0.0316 & 0.753 & 0.0029 & 0.093 \\
\hline 59 & 0.0253 & 0.0952 & 0.265 & 0.0074 & 0.078 \\
\hline
\end{tabular}




\begin{tabular}{|c|c|c|c|c|c|}
\hline 60 & 0.5196 & 0.6627 & 0.784 & 0.0051 & 0.008 \\
\hline 61 & 0.6671 & 1.0105 & 0.660 & 0.0123 & 0.012 \\
\hline 62 & 0.3525 & 0.7832 & 0.450 & 0.0224 & 0.029 \\
\hline 64 & 0.4679 & 0.5596 & 0.836 & 0.0213 & 0.038 \\
\hline 65 & 0.5290 & 0.6387 & 0.828 & 0.0059 & 0.009 \\
\hline 66 & 0.1619 & 0.4265 & 0.380 & 0.0355 & 0.083 \\
\hline 67 & 0.0496 & 0.4413 & 0.112 & 0.0286 & 0.065 \\
\hline 68 & 0.4947 & 0.7064 & 0.700 & 0.0141 & 0.020 \\
\hline 69 & 0.4275 & 0.7465 & 0.573 & 0.0325 & 0.044 \\
\hline 70 & 0.2205 & 0.5005 & 0.441 & 0.0266 & 0.053 \\
\hline 71 & 0.4191 & 0.7599 & 0.551 & 0.0426 & 0.056 \\
\hline 72 & 0.3962 & 0.5325 & 0.744 & 0.0123 & 0.023 \\
\hline 75 & 0.2328 & 0.4077 & 0.571 & 0.0212 & 0.052 \\
\hline 76 & 0.3877 & 0.6277 & 0.618 & 0.0128 & 0.020 \\
\hline 78 & 0.0303 & 0.0588 & 0.514 & 0.0050 & 0.084 \\
\hline 79 & 0.0810 & 0.1166 & 0.695 & 0.0077 & 0.066 \\
\hline 80 & 0.3898 & 0.6561 & 0.594 & 0.0183 & 0.028 \\
\hline 81 & 0.0192 & 0.0701 & 0.274 & 0.0062 & 0.089 \\
\hline 83 & 0.5318 & 0.6035 & 0.881 & 0.0031 & 0.005 \\
\hline 84 & 0.0554 & 0.0845 & 0.656 & 0.0063 & 0.074 \\
\hline 85 & 0.3609 & 0.4398 & 0.821 & 0.0071 & 0.016 \\
\hline 86 & 0.3190 & 0.3252 & 0.981 & 0.0044 & 0.014 \\
\hline 87 & 0.1221 & 0.1878 & 0.650 & 0.0133 & 0.071 \\
\hline 88 & 0.0021 & 0.0023 & 0.919 & 0.0002 & 0.070 \\
\hline 89 & 0.5918 & 0.7099 & 0.834 & 0.0083 & 0.012 \\
\hline 90 & 0.3559 & 0.8405 & 0.423 & 0.0231 & 0.027 \\
\hline 91 & 0.0017 & 0.0610 & 0.027 & 0.0017 & 0.027 \\
\hline 92 & 0.3910 & 0.4834 & 0.809 & 0.0111 & 0.023 \\
\hline 94 & 0.0071 & 0.0134 & 0.529 & 0.0047 & 0.353 \\
\hline 95 & 0.0213 & 0.0215 & 0.988 & 0.0009 & 0.042 \\
\hline 96 & 0.0249 & 0.0398 & 0.626 & 0.0043 & 0.107 \\
\hline 99 & 0.0878 & 0.1059 & 0.828 & 0.0084 & 0.079 \\
\hline 100 & 0.0467 & 0.0695 & 0.673 & 0.0095 & 0.137 \\
\hline 101 & 0.0887 & 0.0976 & 0.910 & 0.0136 & 0.139 \\
\hline 102 & 0.2066 & 0.2363 & 0.875 & 0.0052 & 0.022 \\
\hline 103 & 0.0368 & 0.0581 & 0.634 & 0.0090 & 0.156 \\
\hline 104 & 0.2997 & 0.6710 & 0.447 & 0.0461 & 0.069 \\
\hline 105 & 0.0102 & 0.0157 & 0.651 & 0.0019 & 0.118 \\
\hline 106 & 0.3397 & 0.5144 & 0.660 & 0.0158 & 0.031 \\
\hline 108 & 0.4969 & 0.8539 & 0.582 & 0.0147 & 0.017 \\
\hline 109 & 0.6172 & 0.6988 & 0.883 & 0.0070 & 0.010 \\
\hline 111 & 0.1096 & 0.2954 & 0.371 & 0.0192 & 0.065 \\
\hline 114 & 0.3353 & 0.4885 & 0.686 & 0.0135 & 0.028 \\
\hline 115 & 0.5842 & 0.6999 & 0.835 & 0.0101 & 0.014 \\
\hline 116 & 0.6307 & 0.7512 & 0.840 & 0.0160 & 0.021 \\
\hline 117 & 0.1590 & 0.2094 & 0.759 & 0.0141 & 0.068 \\
\hline 118 & 0.0124 & 0.0328 & 0.379 & 0.0063 & 0.193 \\
\hline 119 & 0.5501 & 0.7534 & 0.730 & 0.0067 & 0.009 \\
\hline 120 & 0.2731 & 0.3184 & 0.858 & 0.0059 & 0.018 \\
\hline 121 & 0.0026 & 0.0085 & 0.299 & 0.0025 & 0.298 \\
\hline 122 & 0.2410 & 0.3178 & 0.758 & 0.0142 & 0.045 \\
\hline 123 & 0.0665 & 0.1021 & 0.652 & 0.0057 & 0.056 \\
\hline 124 & 0.3107 & 0.4108 & 0.756 & 0.0062 & 0.015 \\
\hline 125 & 0.0968 & 0.2097 & 0.461 & 0.0133 & 0.063 \\
\hline 126 & 0.2565 & 0.2661 & 0.964 & 0.0029 & 0.011 \\
\hline 127 & 0.7554 & 0.9614 & 0.786 & 0.0108 & 0.011 \\
\hline
\end{tabular}

S-11 


\begin{tabular}{|c|c|c|c|c|c|}
\hline 128 & 0.2760 & 0.4823 & 0.572 & 0.0214 & 0.044 \\
\hline 131 & 0.5977 & 0.8919 & 0.670 & 0.0222 & 0.025 \\
\hline 132 & 0.3340 & 0.5931 & 0.563 & 0.0185 & 0.031 \\
\hline 133 & 0.0330 & 0.1256 & 0.263 & 0.0047 & 0.038 \\
\hline 135 & 0.5690 & 0.6520 & 0.873 & 0.0056 & 0.009 \\
\hline 136 & 0.0385 & 0.0979 & 0.393 & 0.0168 & 0.172 \\
\hline 137 & 0.5888 & 0.7594 & 0.775 & 0.0130 & 0.017 \\
\hline 138 & 0.0000 & 0.0000 & 1.029 & 0.0000 & 0.261 \\
\hline 139 & 0.0239 & 0.2498 & 0.096 & 0.0095 & 0.038 \\
\hline 140 & 0.6765 & 0.8395 & 0.806 & 0.0148 & 0.018 \\
\hline 141 & 0.4442 & 0.6194 & 0.717 & 0.0080 & 0.013 \\
\hline 142 & 0.0087 & 0.0130 & 0.670 & 0.0018 & 0.138 \\
\hline 143 & 0.3568 & 0.3598 & 0.992 & 0.0010 & 0.003 \\
\hline 144 & 0.6221 & 0.7402 & 0.840 & 0.0102 & 0.014 \\
\hline 145 & 0.0360 & 0.0873 & 0.412 & 0.0045 & 0.051 \\
\hline 147 & 0.3915 & 0.4532 & 0.864 & 0.0063 & 0.014 \\
\hline 148 & 0.0923 & 0.2291 & 0.403 & 0.0111 & 0.048 \\
\hline 149 & 0.0099 & 0.0151 & 0.654 & 0.0048 & 0.317 \\
\hline 150 & 0.0216 & 0.0333 & 0.651 & 0.0049 & 0.146 \\
\hline 151 & 0.1649 & 0.2734 & 0.603 & 0.0215 & 0.079 \\
\hline 152 & 0.0078 & 0.0140 & 0.559 & 0.0046 & 0.327 \\
\hline 153 & 0.0053 & 0.0051 & 1.030 & 0.0009 & 0.172 \\
\hline 154 & 0.3247 & 0.5242 & 0.619 & 0.0124 & 0.024 \\
\hline 155 & 0.0185 & 0.0523 & 0.355 & 0.0071 & 0.135 \\
\hline 157 & 0.0130 & 0.0396 & 0.328 & 0.0052 & 0.132 \\
\hline 158 & 0.2381 & 0.2642 & 0.901 & 0.0042 & 0.016 \\
\hline 159 & 0.6449 & 0.8818 & 0.731 & 0.0078 & 0.009 \\
\hline 161 & 0.0124 & 0.0275 & 0.452 & 0.0021 & 0.077 \\
\hline 162 & 0.6297 & 0.7607 & 0.828 & 0.0046 & 0.006 \\
\hline
\end{tabular}


Table S4: Side chain entropy $S /\left(k_{\mathrm{B}} N_{\chi}\right)$ within the tumbling time $\tau_{\mathrm{R}}$ and in the long-time limit with the CHARMM36 force field. STD and SEM are the standard deviation and the standard error of the mean, respectively.

\begin{tabular}{|c|c|c|c|c|c|}
\hline Residue number & $S /\left(k_{B} N_{\chi}\right)\left[\tau_{\mathbf{R}}\right]$ & $S /\left(k_{B} N_{\chi}\right)\left[t_{\operatorname{sim}}\right]$ & $S\left[\tau_{\mathbf{R}}\right] / S\left[t_{\operatorname{sim}}\right]$ & STD & SEM \\
\hline 1 & 0.4345 & 0.6057 & 0.717 & 0.0148 & 0.024 \\
\hline 2 & 0.2841 & 0.4044 & 0.702 & 0.0242 & 0.060 \\
\hline 3 & 0.1362 & 0.2186 & 0.623 & 0.0289 & 0.132 \\
\hline 4 & 0.1295 & 0.4621 & 0.280 & 0.0121 & 0.026 \\
\hline 5 & 0.5129 & 0.6645 & 0.772 & 0.0135 & 0.020 \\
\hline 6 & 0.4274 & 0.6138 & 0.696 & 0.0225 & 0.037 \\
\hline 7 & 0.0370 & 0.0637 & 0.580 & 0.0078 & 0.123 \\
\hline 8 & 0.1412 & 0.6591 & 0.214 & 0.0256 & 0.039 \\
\hline 9 & 0.4565 & 0.7447 & 0.613 & 0.0101 & 0.014 \\
\hline 10 & 0.2321 & 0.8071 & 0.288 & 0.0354 & 0.044 \\
\hline 11 & 0.2801 & 0.6396 & 0.438 & 0.0172 & 0.027 \\
\hline 13 & 0.1265 & 0.2039 & 0.620 & 0.0247 & 0.121 \\
\hline 14 & 0.0571 & 0.3695 & 0.154 & 0.0070 & 0.019 \\
\hline 15 & 0.0495 & 0.0704 & 0.703 & 0.0051 & 0.072 \\
\hline 16 & 0.6102 & 0.7283 & 0.838 & 0.0061 & 0.008 \\
\hline 17 & 0.2288 & 0.3887 & 0.589 & 0.0260 & 0.067 \\
\hline 18 & 0.0653 & 0.4938 & 0.132 & 0.0234 & 0.047 \\
\hline 19 & 0.4240 & 0.5188 & 0.817 & 0.0205 & 0.039 \\
\hline 20 & 0.2755 & 0.5393 & 0.511 & 0.0146 & 0.027 \\
\hline 21 & 0.6143 & 1.0032 & 0.612 & 0.0806 & 0.080 \\
\hline 22 & 0.4426 & 0.5469 & 0.809 & 0.0184 & 0.034 \\
\hline 24 & 0.2053 & 0.4128 & 0.497 & 0.0226 & 0.055 \\
\hline 25 & 0.3166 & 0.3357 & 0.943 & 0.0092 & 0.028 \\
\hline 26 & 0.1328 & 0.7821 & 0.170 & 0.0343 & 0.044 \\
\hline 27 & 0.2959 & 0.3406 & 0.869 & 0.0104 & 0.030 \\
\hline 29 & 0.2381 & 0.4617 & 0.516 & 0.0157 & 0.034 \\
\hline 31 & 0.0260 & 0.0313 & 0.829 & 0.0060 & 0.192 \\
\hline 32 & 0.3450 & 0.4111 & 0.839 & 0.0264 & 0.064 \\
\hline 33 & 0.0029 & 0.0256 & 0.114 & 0.0020 & 0.077 \\
\hline 34 & 0.0002 & 0.0006 & 0.334 & 0.0002 & 0.323 \\
\hline 35 & 0.4268 & 0.4902 & 0.871 & 0.0070 & 0.014 \\
\hline 36 & 0.2141 & 0.5112 & 0.419 & 0.0276 & 0.054 \\
\hline 37 & 0.1704 & 0.1747 & 0.976 & 0.0062 & 0.035 \\
\hline 38 & 0.0117 & 0.0239 & 0.490 & 0.0049 & 0.205 \\
\hline 39 & 0.0357 & 0.0458 & 0.780 & 0.0027 & 0.060 \\
\hline 40 & 0.5524 & 0.6161 & 0.897 & 0.0105 & 0.017 \\
\hline 43 & 0.2891 & 0.3400 & 0.850 & 0.0032 & 0.009 \\
\hline 44 & 0.4778 & 0.8649 & 0.552 & 0.0265 & 0.031 \\
\hline 45 & 0.3154 & 0.3576 & 0.882 & 0.0050 & 0.014 \\
\hline 46 & -0.0000 & 0.0000 & -0.000 & 0.0000 & 0.000 \\
\hline 47 & 0.2902 & 0.2951 & 0.983 & 0.0076 & 0.026 \\
\hline 48 & 0.4021 & 0.5447 & 0.738 & 0.0061 & 0.011 \\
\hline 50 & 0.0412 & 0.0733 & 0.563 & 0.0103 & 0.140 \\
\hline 52 & 0.0863 & 0.1258 & 0.686 & 0.0085 & 0.068 \\
\hline 53 & 0.4740 & 0.7887 & 0.601 & 0.0210 & 0.027 \\
\hline 54 & 0.0010 & 0.1892 & 0.005 & 0.0005 & 0.003 \\
\hline 55 & 0.5919 & 0.6549 & 0.904 & 0.0115 & 0.018 \\
\hline 57 & 0.3711 & 0.7718 & 0.481 & 0.0264 & 0.034 \\
\hline 58 & 0.0339 & 0.0355 & 0.954 & 0.0024 & 0.068 \\
\hline 59 & 0.0192 & 0.0520 & 0.369 & 0.0129 & 0.248 \\
\hline
\end{tabular}




\begin{tabular}{|c|c|c|c|c|c|}
\hline 60 & 0.3788 & 0.6734 & 0.563 & 0.0232 & 0.035 \\
\hline 61 & 0.5404 & 0.6133 & 0.881 & 0.0105 & 0.017 \\
\hline 62 & 0.0431 & 0.2439 & 0.177 & 0.0109 & 0.045 \\
\hline 64 & 0.4996 & 0.6455 & 0.774 & 0.0116 & 0.018 \\
\hline 65 & 0.5755 & 0.7568 & 0.761 & 0.0101 & 0.013 \\
\hline 66 & 0.1012 & 0.3644 & 0.278 & 0.0149 & 0.041 \\
\hline 67 & 0.2982 & 0.4300 & 0.693 & 0.0180 & 0.042 \\
\hline 68 & 0.4759 & 0.5336 & 0.892 & 0.0150 & 0.028 \\
\hline 69 & 0.3849 & 0.5600 & 0.687 & 0.0091 & 0.016 \\
\hline 70 & 0.1542 & 0.3751 & 0.411 & 0.0082 & 0.022 \\
\hline 71 & 0.0141 & 0.0267 & 0.527 & 0.0067 & 0.252 \\
\hline 72 & 0.5181 & 0.6156 & 0.842 & 0.0087 & 0.014 \\
\hline 75 & 0.1237 & 0.1963 & 0.630 & 0.0122 & 0.062 \\
\hline 76 & 0.3865 & 0.6145 & 0.629 & 0.0083 & 0.014 \\
\hline 78 & 0.0659 & 0.0759 & 0.868 & 0.0098 & 0.129 \\
\hline 79 & 0.1297 & 0.1564 & 0.829 & 0.0136 & 0.087 \\
\hline 80 & 0.1637 & 0.3044 & 0.538 & 0.0125 & 0.041 \\
\hline 81 & 0.0337 & 0.0783 & 0.430 & 0.0051 & 0.066 \\
\hline 83 & 0.4967 & 0.5690 & 0.873 & 0.0038 & 0.007 \\
\hline 84 & 0.0135 & 0.0173 & 0.778 & 0.0039 & 0.226 \\
\hline 85 & 0.1248 & 0.1946 & 0.641 & 0.0132 & 0.068 \\
\hline 86 & 0.3470 & 0.3490 & 0.994 & 0.0017 & 0.005 \\
\hline 87 & 0.0527 & 0.0954 & 0.552 & 0.0138 & 0.144 \\
\hline 88 & 0.1968 & 0.2146 & 0.917 & 0.0121 & 0.057 \\
\hline 89 & 0.1891 & 0.5115 & 0.370 & 0.0097 & 0.019 \\
\hline 90 & 0.2922 & 0.9783 & 0.299 & 0.0272 & 0.028 \\
\hline 91 & 0.0022 & 0.0163 & 0.135 & 0.0022 & 0.135 \\
\hline 92 & 0.5296 & 0.6064 & 0.873 & 0.0065 & 0.011 \\
\hline 94 & 0.0104 & 0.0257 & 0.406 & 0.0051 & 0.198 \\
\hline 95 & 0.1095 & 0.1213 & 0.902 & 0.0051 & 0.042 \\
\hline 96 & 0.0512 & 0.1782 & 0.287 & 0.0154 & 0.086 \\
\hline 99 & 0.0243 & 0.0303 & 0.802 & 0.0018 & 0.060 \\
\hline 100 & 0.0072 & 0.0128 & 0.562 & 0.0020 & 0.155 \\
\hline 101 & 0.3075 & 0.4004 & 0.768 & 0.0125 & 0.031 \\
\hline 102 & 0.3238 & 0.4206 & 0.770 & 0.0132 & 0.031 \\
\hline 103 & 0.0031 & 0.0046 & 0.676 & 0.0009 & 0.184 \\
\hline 104 & 0.0681 & 0.2971 & 0.229 & 0.0135 & 0.046 \\
\hline 105 & 0.0920 & 0.6098 & 0.151 & 0.0230 & 0.038 \\
\hline 106 & 0.3481 & 0.5440 & 0.640 & 0.0110 & 0.020 \\
\hline 108 & 0.3372 & 0.6890 & 0.489 & 0.0260 & 0.038 \\
\hline 109 & 0.7495 & 0.8235 & 0.910 & 0.0141 & 0.017 \\
\hline 111 & 0.0392 & 0.2469 & 0.159 & 0.0145 & 0.059 \\
\hline 114 & 0.4394 & 0.5391 & 0.815 & 0.0152 & 0.028 \\
\hline 115 & 0.5721 & 0.7138 & 0.801 & 0.0136 & 0.019 \\
\hline 116 & 0.6503 & 0.7780 & 0.836 & 0.0111 & 0.014 \\
\hline 117 & 0.1443 & 0.3324 & 0.434 & 0.0269 & 0.081 \\
\hline 118 & 0.0201 & 0.0533 & 0.378 & 0.0107 & 0.201 \\
\hline 119 & 0.2805 & 0.6302 & 0.445 & 0.0245 & 0.039 \\
\hline 120 & 0.2871 & 0.3661 & 0.784 & 0.0091 & 0.025 \\
\hline 121 & 0.0069 & 0.0206 & 0.333 & 0.0028 & 0.138 \\
\hline 122 & 0.5202 & 0.6226 & 0.836 & 0.0101 & 0.016 \\
\hline 123 & 0.1899 & 0.3840 & 0.495 & 0.0115 & 0.030 \\
\hline 124 & 0.3418 & 0.4962 & 0.689 & 0.0125 & 0.025 \\
\hline 125 & 0.0704 & 0.2593 & 0.272 & 0.0134 & 0.052 \\
\hline 126 & 0.3325 & 0.3368 & 0.987 & 0.0023 & 0.007 \\
\hline 127 & 0.5034 & 0.6053 & 0.832 & 0.0101 & 0.017 \\
\hline
\end{tabular}




\begin{tabular}{|c|c|c|c|c|c|}
\hline 128 & 0.3059 & 0.4992 & 0.613 & 0.0426 & 0.085 \\
\hline 131 & 0.3306 & 0.7753 & 0.426 & 0.0247 & 0.032 \\
\hline 132 & 0.3708 & 0.4585 & 0.809 & 0.0148 & 0.032 \\
\hline 133 & 0.0481 & 0.1690 & 0.285 & 0.0136 & 0.080 \\
\hline 135 & 0.5382 & 0.6481 & 0.830 & 0.0064 & 0.010 \\
\hline 136 & 0.0052 & 0.0231 & 0.225 & 0.0052 & 0.225 \\
\hline 137 & 0.2377 & 0.4936 & 0.481 & 0.0147 & 0.030 \\
\hline 138 & 0.0004 & 0.0004 & 0.805 & 0.0001 & 0.204 \\
\hline 139 & 0.0273 & 0.2257 & 0.121 & 0.0084 & 0.037 \\
\hline 140 & 0.6323 & 0.7427 & 0.851 & 0.0108 & 0.014 \\
\hline 141 & 0.1450 & 0.2647 & 0.548 & 0.0198 & 0.075 \\
\hline 142 & 0.0402 & 0.0394 & 1.020 & 0.0081 & 0.205 \\
\hline 143 & 0.1874 & 0.1895 & 0.989 & 0.0051 & 0.027 \\
\hline 144 & 0.5120 & 0.6025 & 0.850 & 0.0223 & 0.037 \\
\hline 145 & 0.0677 & 0.4466 & 0.152 & 0.0143 & 0.032 \\
\hline 147 & 0.3696 & 0.4794 & 0.771 & 0.0125 & 0.026 \\
\hline 148 & 0.1099 & 0.3653 & 0.301 & 0.0135 & 0.037 \\
\hline 149 & 0.0154 & 0.0256 & 0.602 & 0.0082 & 0.320 \\
\hline 150 & 0.1137 & 0.1438 & 0.791 & 0.0093 & 0.065 \\
\hline 151 & 0.2037 & 0.3567 & 0.571 & 0.0267 & 0.075 \\
\hline 152 & 0.0118 & 0.0414 & 0.285 & 0.0077 & 0.185 \\
\hline 153 & 0.2762 & 0.3716 & 0.743 & 0.0102 & 0.027 \\
\hline 154 & 0.3002 & 0.4842 & 0.620 & 0.0084 & 0.017 \\
\hline 155 & 0.0290 & 0.0595 & 0.487 & 0.0047 & 0.079 \\
\hline 157 & 0.0228 & 0.6068 & 0.038 & 0.0107 & 0.018 \\
\hline 158 & 0.1324 & 0.2555 & 0.518 & 0.0103 & 0.040 \\
\hline 159 & 0.3963 & 0.4919 & 0.806 & 0.0109 & 0.022 \\
\hline 161 & 0.0057 & 0.0324 & 0.176 & 0.0026 & 0.082 \\
\hline 162 & 0.4108 & 0.5811 & 0.707 & 0.0127 & 0.022 \\
\hline
\end{tabular}

